\title{
Particle motion in Stokes flow near a plane fluid-fluid interface. Part 2. Linear shear and axisymmetric straining flows
}

\author{
By SEUNG-MAN YANG AND L. GARY LEAL \\ Department of Chemical Engineering, California Institute of Technology, \\ Pasadena, California 91125
}

(Received 14 November 1983 and in revised form 27 June 1984)

\begin{abstract}
We consider the motion of a sphere or a slender body in the presence of a plane fluid-fluid interface with an arbitrary viscosity ratio, when the fluids undergo a linear undisturbed flow. First, the hydrodynamic relationships for the force and torque on the particle at rest in the undisturbed flow field are determined, using the method of reflections, from the spatial distribution of Stokeslets, rotlets and higher-order singularities in Stokes flow. These fundamental relationships are then applied, in combination with the corresponding solutions obtained in earlier publications for the translation and rotation through a quiescent fluid, to determine the motion of a neutrally buoyant particle freely suspended in the flow. The theory yields general trajectory equations for an arbitrary viscosity ratio which are in good agreement with both exact-solution results and experimental data for sphere motions near a rigid plane wall. Among the most interesting results for motion of slender bodies is the generalization of the Jeffrey orbit equations for linear simple shear flow.
\end{abstract}

\section{Introduction}

In this paper we consider the creeping motion of a sphere or a slender body in linear shear and axisymmetric straining flows near a plane fluid-fluid interface. Previously, we considered translation and rotation of a sphere when the fluids are at rest at infinity (Lee, Chadwick \& Leal 1979; Lee \& Leal 1980), and, in Part I of the present pair of papers, the same problem was solved for a rigid, straight slender body (Yang \& Leal 1983). Although the quiescent-fluid problem is of some intrinsic interest, and is a logical starting point for investigation of particle motions near a fluid interface, many problems of practical significance involve particle motions in a mean flow at infinity (cf. Goldman, Cox \& Brenner, $1967 a, b$; Goren \& O'Neill 1971 ; Spielman 1977; and references therein). This is true of boundary effects in the rheology of dilute suspensions, theories of Brownian motion near a phase boundary and the development of trajectory equations to model the 'collection' of very small particles at the surface of larger bubbles or drops in flotation processes (cf. Dukhin \& Rulev 1977).

The majority of previous analyses of creeping particle motion near a flat wall or interface were restricted to spherical particles, and utilized separation of variables in bipolar coordinates; cf. Jeffery (1912), Brenner (1961) and Lee \& Leal (1980) for motion in a quiescent flow. Goren \& O'Neill (1971) used the same approach to consider the motion of a sphere in simple shear flow near a solid, plane wall, and, more recently, Dukhin \& Rulev (1977) considered a sphere on the axis of symmetry of a pure straining flow near a gas-liquid interface. An alternative approach, which is essential 
if the particles are not spherical, is to construct solutions using spatial distributions of fundamental singularities. This approach has been known since the pioneering work of Lorentz (1907). Recently, fundamental solutions were developed for a point force and higher-order singularities near a fluid-fluid interface by a generalization of the Lorentz analysis, and used to solve for the creeping motion of a spherical particle when the fluids are at rest at infinity (Lee et al. 1979). The same basic method has also been applied, in a slender-body approximation, to investigate the translation and rotation of a straight, rigid slender body through a quiescent fluid (Fulford \& Blake (1983) for translation with the particle axis either perpendicular or parallel to the interface; Yang \& Leal (1983) for translation and rotation with an arbitrary orientation).

In this present work we use the singularity method to study the hydrodynamic interactions between either a sphere or a straight, rigid slender body and a flat fluid-fluid interface in linear flows that are compatible with the presence of a plane interface. The solutions we obtain provide the hydrodynamic 'resistance' tensors that define the relationships between the force and torque on the particle at rest in the flow field, the undisturbed flow parameters such as strain rate or shear rate, and the translational and angular velocities of the particle. These fundamental relationships are then used to calculate the particle trajectories in simple shear and axisymmetric straining flows.

\section{Basic equations}

We begin by considering the governing equations and boundary conditions for a rigid particle (i.e. sphere or slender body) at rest near a flat fluid-fluid interface of two immiscible fluids 1 and 2. The particle is assumed to be in fluid 2, and the undisturbed velocity field is given in the form:

or

$$
\sigma_{i}=E \cdot x \text { for pure straining flow }
$$

in which $\lambda\left(=\mu_{1} / \mu_{2}\right)$ is the viscosity ratio of the two fluids, $\sigma_{i}$ is the undisturbed velocity field in fluid $i(=1,2)$, and $\boldsymbol{x}$ denotes a position vector measured from an origin that is placed at the interface. These undisturbed flow fields are depieted in figures $1(a, d)$, and are consistent with the existence of a flat interface at which the normal components of velocities are identically zero (i.e. $\boldsymbol{U} \cdot \boldsymbol{n}=\mathbf{0}$ ). The linear operator $\boldsymbol{E}$ for an axisymmetric extensional flow takes the form

$$
\boldsymbol{E}=\left[\begin{array}{ccc}
E & 0 & 0 \\
0 & E & 0 \\
0 & 0 & -2 E
\end{array}\right]
$$

while that for a linear simple shear flow parallel to the interface is

$$
\boldsymbol{\Gamma}=\left[\begin{array}{ccc}
0 & 0 & \Gamma_{13} \\
0 & 0 & \Gamma_{23} \\
0 & 0 & 0
\end{array}\right]
$$

Here, $E$ and $\Gamma_{j 3}(j=1,2)$ are usually denoted as the strain rate and shear rate respectively. 


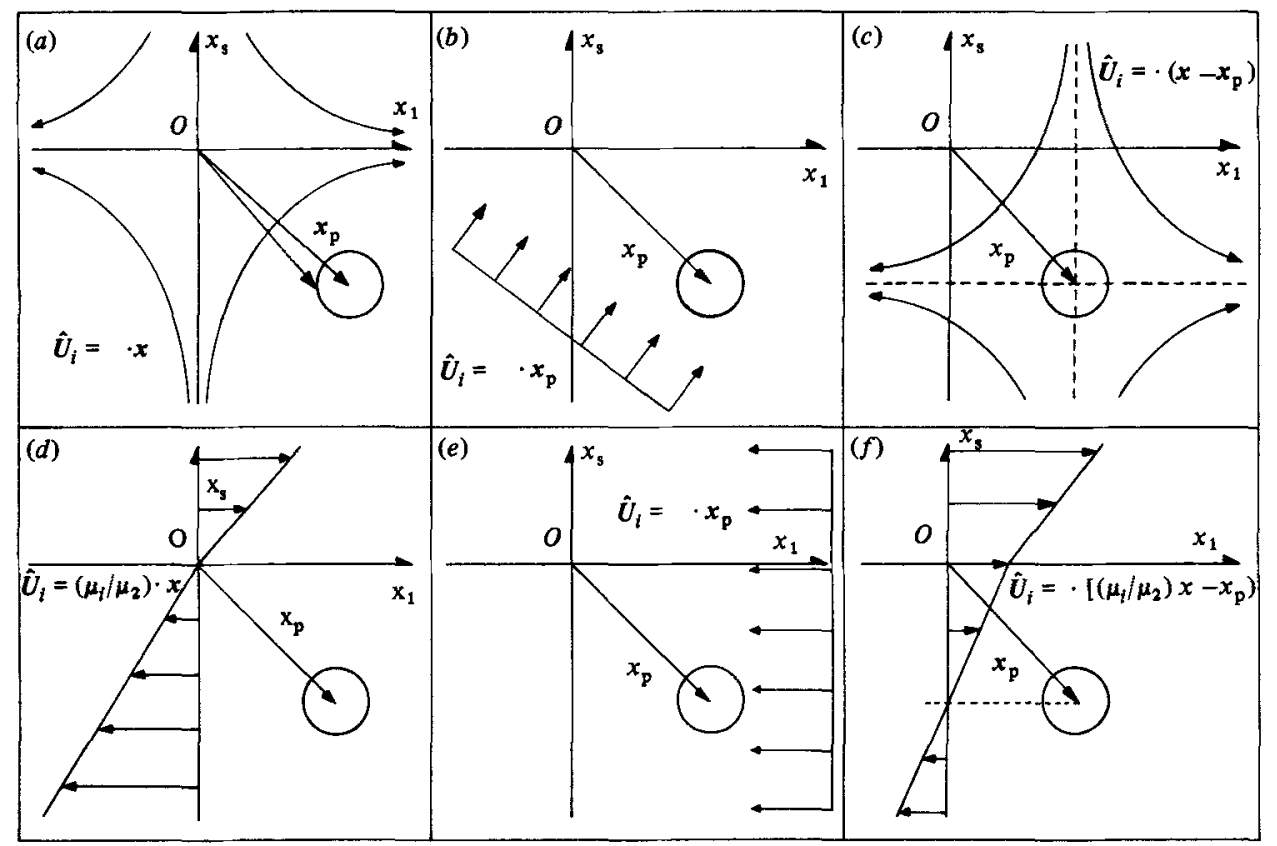

FIGURE 1. Coordinate system with an interface in the $\left(x_{1}, x_{2}\right)$-plane and description of decomposed problems: $(a)$ a sphere in the pure straining flow $\hat{O}_{i}=\boldsymbol{E} \cdot \boldsymbol{x} ;(b)$ a uniform streaming flow $\hat{U}_{i}=\boldsymbol{E} \cdot \boldsymbol{x}_{\mathrm{p}}$ past a stationary sphere at $x_{p} ;(c)$ a sphere at the stagnation point $x_{p}$ of the pure straining flow $\hat{O}_{i}=\boldsymbol{E} \cdot\left(\boldsymbol{x}-\boldsymbol{x}_{\mathrm{p}}\right) ;(d)$ a sphere in the simple shear flow $\hat{O}_{i}=\left(\mu_{i} / \mu_{2}\right) \boldsymbol{\Gamma} \cdot \boldsymbol{x} ;(e)$ a uniform streaming flow $\sigma_{i}=\Gamma \cdot x_{\mathrm{p}}$ past a stationary sphere; $(f)$ a sphere at the stagnation point $x_{\mathrm{p}}$ of the simple shear flow $\hat{O}_{i}=\Gamma \cdot\left[\left(\mu_{i} / \mu_{2}\right) x-x_{\mathrm{p}}\right]$.

In the present problem the Reynolds number is defined by

$$
R e=\frac{E l_{\mathrm{c}}^{2}}{\nu_{2}} \quad\left(\text { or } \frac{\Gamma_{j 3} l_{\mathrm{c}}^{2}}{\nu_{2}}\right),
$$

where $l_{\mathrm{c}}$ is a characteristic lengthscale of the particle (i.e. either the sphere radius $a$ or the half-length $l$ of the slender body), and $v_{2}$ represents the kinematic viscosity of fluid 2. We assume that the Reynolds number is sufficiently small (i.e. $R e \ll 1$ ) that the motion is quasi-steady and the creeping motion approximation applicable. The equations of motion therefore reduce to steady Stokes equations in both fluids. Further, the linearity of the Stokes equation enables us to decompose the undisturbed flow field $\hat{O}_{i}=L_{i} \cdot x$ into a constant vector (i.e. a uniform streaming flow, figures $1 b, e$ ),

$$
O_{i}=L_{2} \cdot x_{\mathrm{p}}
$$

and a linear part with vanishing velocity at the body centre (i.e. figures $1 c, f$ ),

$$
\hat{O}_{i}=L_{i} \cdot x-L_{2} \cdot x_{\mathrm{p}}
$$

Here $L_{i}$ denotes either the strain-rate tensor $\boldsymbol{E}$ in each fluid, or shear-rate tensors $\left(\mu_{i} / \mu_{2}\right) \Gamma$ for $i=1$ and 2 . The Stokes' problem for $U_{i}=L_{2} \cdot x_{\mathrm{p}}$ of $(2 a)$, which is simply a translation of the fluid system including the interface past a stationary particle, is precisely equivalent to the problem of particle translation with velocity $-\boldsymbol{L}_{2} \cdot \boldsymbol{x}_{\mathrm{p}}$ through a quiescent fluid with stationary interface (cf. figures $1 b, e$ ). A complete detailed solution is available for this problem for both a sphere and a slender body 
from Lee et al. (1979) and Yang \& Leal (1983), who determined the relationship between the hydrodynamic force $F$ and torque $T$ on the body and the translational velocity

$$
F=-K_{\mathrm{T}} \cdot \boldsymbol{L}_{\mathbf{2}} \cdot \boldsymbol{x}_{\mathrm{p}}, \quad \boldsymbol{T}=-\boldsymbol{K}_{\mathrm{C}} \cdot \boldsymbol{L}_{2} \cdot \boldsymbol{x}_{\mathrm{p}}
$$

where $\boldsymbol{K}_{\mathrm{T}}$ and $\boldsymbol{\kappa}_{\mathrm{C}}$ denote the translational and coupling tensors respectively.

It thus remains only to solve the problem for the linear undisturbed flow $\sigma_{i}=L_{i} \cdot x-L_{2} \cdot x_{\mathrm{p}}$ with $\hat{O}_{2}=0$ at the body centre (cf. figures $1 c, f$ ). We define, for convenience, a disturbance velocity field $u$ as the difference between the actual velocity $\hat{\boldsymbol{u}}$ in the presence of the particle and the undisturbed flow, i.e.

$$
\boldsymbol{u}_{i}=\hat{\boldsymbol{u}}_{i}-\left\{\boldsymbol{L}_{\boldsymbol{i}} \cdot \boldsymbol{x}-\boldsymbol{L}_{2} \cdot \boldsymbol{x}_{\mathrm{p}}\right\} \quad(i=1,2) .
$$

The equations of motion for the disturbance velocity field are

$$
\boldsymbol{\nabla} p_{i}=\frac{\mu_{i}}{\mu_{2}} \nabla^{2} \boldsymbol{u}_{i}, \quad \boldsymbol{\nabla} \cdot \boldsymbol{u}_{i}=\mathbf{0} \quad(i=1,2),
$$

in which the variables may be considered to be non-dimensionalized with respect to the characteristic variables $l_{\mathrm{c}}=a$ (or $\left.l\right), t_{\mathrm{c}}=1 / E\left(\right.$ or $\left.1 / \Gamma_{j 3}\right)$ and $p_{\mathrm{c}}=\mu_{2} E$ (or $\mu_{2} \Gamma_{j 3}$ ). Thus, for the following analysis, the non-dimensionalized variables such as the hydrodynamic force $\boldsymbol{F}$ and torque $\boldsymbol{T}$, and the translational and angular velocities of the body, $U$ and $\boldsymbol{\Omega}$, are based on the corresponding characteristic variables, i.e. $F_{\mathrm{c}}=\mu_{2} E l_{\mathrm{c}}^{2}\left(\right.$ or $\left.\mu_{2} \Gamma_{j 3} l_{\mathrm{c}}^{2}\right), T_{\mathrm{c}}=\mu_{2} E l_{\mathrm{c}}^{3}\left(\right.$ or $\mu_{2} \Gamma_{j 3} l_{\mathrm{c}}^{3}$ ), $U_{\mathrm{c}}=E l_{\mathrm{c}}$ (or $\Gamma_{j 3} l_{\mathrm{c}}$ ) and $\Omega_{\mathrm{c}}=E$ (or $\Gamma_{j 3}$ ) respectively. The boundary conditions for $u_{1}$ and $u_{2}$ in this disturbance-flow problem are

$$
\begin{gathered}
\boldsymbol{u}_{1}, \boldsymbol{u}_{2} \rightarrow \mathbf{0} \text { as }|x| \rightarrow \infty, \\
\boldsymbol{u}_{2}=-\boldsymbol{L}_{2} \cdot\left(\boldsymbol{x}_{\mathrm{B}}-\boldsymbol{x}_{\mathrm{p}}\right) \quad \text { on } \boldsymbol{x}_{\mathrm{B}} \in S_{\mathrm{p}},
\end{gathered}
$$

plus the interface conditions (i.e. continuity of velocity and tangential stress and zero normal velocity). In $(5 b) x_{\mathrm{B}}$ denotes a point on the body surface $S_{\mathrm{p}}$. From the point of view of (4) and (5), the problem is seen to be exactly the same as if a velocity field $u_{2}\left(x_{B}\right)=-L_{2} \cdot\left(x_{B}-x_{p}\right)$ is generated at the surface of a body that is near a flat fluid-fluid interface in a fluid at rest at infinity.

For a spherical particle we consider the asymptotic limit

$$
\delta=\frac{a}{d} \ll 1
$$

in which $d$ is a separation distance between the sphere centre and the interface. In this case, the singularity method can be reduced to the superposition of fundamental solutions for a point force $\boldsymbol{a}$ (i.e. Stokeslet), a potential dipole $\boldsymbol{\beta}$ and higher-order singularities (e.g. a stresslet, a rotlet, a potential quadrupole, etc.) at the sphere centre. Fundamental solutions of the creeping-motion equation for a point force (and higher-order singularities) can be obtained easily from the corresponding solntions in an unbounded fluid by following the prescription of Lee et al. (1979). The fundamental solutions automatically satisfy the conditions of velocity and stress continuity, as well as zero normal velocity at a flat fluid interface, plus the boundary condition ( $5 a)$ of vanishing velocity in the far field (cf. Lee et al. 1979). All that remains is to determine the combination of these singularities at the sphere centre $x_{\mathrm{p}}$ that satisfies the boundary condition $(5 b)$. In particular, we must determine the densities and orientations of these singularities so that the disturbance velocity $u_{2}(x)$ is at least approximately equal to $-L_{2} \cdot\left(x_{B}-x_{p}\right)$ at all points of the sphere surface.

For a slender body the problem of particle motion near an interface (i.e. the disturbance-flow problem) (4) and (5) can be solved using the slender-body theory 
of low-Reynolds-number flow (Batchelor 1970; Fulford \& Blake 1983; Yang \& Leal 1983 ; and others). In this approach, the disturbance flow produced by the body is approximated by a line distribution of Stokeslets and potential dipoles along the body centreline (rather than a superposition of higher-order singularities at one point $\boldsymbol{x}_{\mathrm{p}}$ or a surface distribution of Stokeslets), and the orientation and strength of these singularities are determined in order to satisfy the boundary condition $(5 b)$ to an order of approximation $O\left(\epsilon^{2}\right)$, where $\epsilon=(\ln 2 \kappa)^{-1}$ and $\kappa$ is the axis ratio of the slender body.

The complete solution for a particle located at arbitrary point $x_{p}$ in a linear flow field $\hat{U}_{i}=L_{i} \cdot x$ near a flat interface is obtained by superposition of the corresponding solution for the linear flow $\hat{O}_{i}=\boldsymbol{L}_{i} \cdot \boldsymbol{x}-\boldsymbol{L}_{2} \cdot \boldsymbol{x}_{\mathrm{p}}$, with $\boldsymbol{U}_{2}=\mathbf{0}$ at the body centre, and the solution (i.e. $(3 a, b))$ for the uniform streaming flow $\hat{O}_{i}=L_{2} \cdot x_{\mathrm{p}}$. In the theoretical analysis that follows, we consider the hydrodynamic force and torque acting on a stationary particle (sphere or slender body) in the presence of both an axisymmetric uniaxial extensional flow and a linear shear flow. These results are then used in $\$ 5$ to calculate the trajectories of a freely suspended sphere or slender body in the same flows near a fluid-fluid interface.

\section{Solutions for a spherical particle}

\subsection{Pure straining flow}

We begin with the creeping motion of a fluid in the vicinity of a stationary spherical particle that is located at an arbitrary point $x_{\mathrm{p}}=\left(x_{\mathrm{p} 1}, x_{\mathrm{p} 2},-d\right)$ in fluid 2 when the undisturbed motion is an axisymmetric uniaxial straining flow $(1 a)$ with origin at the particle centre. Here we utilize the disturbance-flow formulation defined by (4) and (5), and consider only the limit $\delta \equiv a / d \ll 1$.

Since $\delta \ll 1$, the most convenient solution technique is the method of reflections, as was also used and explained in some detail by Lee et al. (1979) for the uniformstreaming problem. The zeroth-order approximation in this procedure $\left(\boldsymbol{u}_{2}^{(0)}, p_{2}^{(0)}\right)$ is the single-fluid unbounded-domain solution which satisfies boundary conditions exactly at the sphere surface. The problem of a spbere in an axisymmetric straining flow, $\tilde{U}=\boldsymbol{E} \cdot\left(\boldsymbol{x}-\boldsymbol{x}_{\mathrm{p}}\right)$ for an unbounded single fluid was solved by Chwang \&Wu (1975), who showed that a potential quadrupole $(\sigma, v)$ and a stresslet $(\rho, \mu)$ of the forms

$$
\begin{aligned}
\text { stresslet } & (\boldsymbol{\rho}, \mu)=\left(\frac{5}{2} e_{3}, e_{3}\right), \\
\text { potential quadrupole } & (\sigma, v)=\left(\frac{1}{2} e_{3}, e_{3}\right)
\end{aligned}
$$

were required at the sphere centre to satisfy the boundary condition $(5 b)$ at the sphere surface. Thus the zeroth-order (i.e. unbounded single-fluid) solution in the methodof-reflections expansion can be written as

$$
\begin{aligned}
u_{2, \mathrm{EX}}^{(0)}(x) & =\frac{1}{2}\left[\frac{\partial}{\partial x_{3}} u_{\mathrm{D}}\left(x, x_{\mathrm{p}} ; \boldsymbol{e}_{3}\right)+5 u_{\mathrm{SS}}\left(x, x_{\mathrm{p}} ; e_{3}, e_{3}\right)\right], \\
p_{2}^{(0)}(x) & =\frac{5}{2} p_{\mathrm{SS}}\left(x, x_{\mathrm{p}} ; \boldsymbol{e}_{3}, \boldsymbol{e}_{3}\right) .
\end{aligned}
$$

Here $\boldsymbol{u}_{\mathrm{D}}\left(\boldsymbol{x}, \boldsymbol{x}_{\mathrm{p}} ; \boldsymbol{\beta}\right), \boldsymbol{u}_{\mathrm{SS}}\left(\boldsymbol{x}, \boldsymbol{x}_{\mathrm{p}} ; \boldsymbol{\rho}, \boldsymbol{\mu}\right)$ and $p_{\mathrm{SS}}\left(\boldsymbol{x}, \boldsymbol{x}_{\mathrm{p}} ; \boldsymbol{\rho}, \boldsymbol{\mu}\right)$ denote the fundamental solutions for a potential dipole $\beta$ and a stresslet $(\boldsymbol{\rho}, \mu)$ located at $x_{\mathrm{p}}$ in an unbounded fluid, ef. Chwang \& $\mathrm{Wu}(\mathbf{1 9 7 5})$.

Though $\boldsymbol{u}_{2, \mathrm{EX}}^{(0)}(\boldsymbol{x})$ of $(6 a)$ exactly satisfies the boundary condition

$$
u_{2}\left(x_{\mathrm{B}}\right)=-E \cdot\left(x_{\mathrm{B}}-x_{\mathrm{p}}\right)
$$

at all points on the sphere surface, it does not satisfy the conditions at the flat interface. However, Lee et al. (1979) have already shown that in the reflections method 
the first correction $\left(\boldsymbol{u}_{2}^{(1)}, p_{2}^{(1)}\right)$ for the presence of the interface can always be obtained by simply utilizing the same form $(6 a, b)$ as in the zeroth-order solution, but with the fundamental solutions $\boldsymbol{u}_{\mathrm{D}}, \boldsymbol{u}_{\mathrm{SS}}$ and $p_{\mathrm{SS}}$ (for an unbounded fluid) replaced by the corresponding fundamental solutions in the presence of the flat interface, obtained by the simple transformation rule of Lee et al. This yields $\left(\boldsymbol{u}_{2}^{(0)}+\boldsymbol{u}_{2}^{(1)}, p_{2}^{(0)}+p_{2}^{(1)}\right)$. The first 'wall correction' can then be obtained by subtracting the zeroth-order solution $\left(u_{2}^{(0)}, p_{2}^{(0)}\right)$ :

$$
\begin{aligned}
& u_{2}^{(1)}(x)=\frac{1}{2}\left[\frac{\partial}{\partial x_{3}}\left\{u_{2, \mathrm{D}}\left(x, x_{\mathrm{p}} ; e_{3}\right)-u_{\mathrm{D}}\left(x, x_{\mathrm{p}} ; \boldsymbol{e}_{3}\right)\right\}\right. \\
& \left.+5\left\{u_{2, \mathrm{ss}}\left(x, x_{\mathrm{p}} ; e_{3}, e_{3}\right)-u_{\mathrm{SS}}\left(x, x_{\mathrm{p}} ; e_{3}, e_{3}\right)\right\}\right] \text {, } \\
& p_{2}^{(1)}(\boldsymbol{x})=\frac{1}{2}\left[\frac{\partial}{\partial x_{3}} p_{2, \mathrm{D}}\left(\boldsymbol{x}, \boldsymbol{x}_{\mathrm{p}} ; \boldsymbol{e}_{3}\right)+5\left\{p_{2, \mathrm{SS}}\left(\boldsymbol{x}, \boldsymbol{x}_{\mathrm{p}} ; \boldsymbol{e}_{3}, \boldsymbol{e}_{3}\right)-p_{\mathrm{SS}}\left(\boldsymbol{x}, \boldsymbol{x}_{\mathrm{p}} ; \boldsymbol{e}_{3}, \boldsymbol{e}_{3}\right)\right\}\right],
\end{aligned}
$$

where we have denoted the resulting fundamental solutions in the presence of the interface as $u_{2, \mathrm{D}}$ and $\boldsymbol{u}_{2, \mathrm{ss}}$ respectively. Although the combined solution $\left(\boldsymbol{u}_{2}^{(0)}+\boldsymbol{u}_{2}^{(1)}, p_{2}^{(0)}+p_{2}^{(1)}\right)$ satisfies the interface boundary conditions, it now does not satisfy the condition $u_{2}\left(x_{\mathrm{B}}\right)=-\boldsymbol{E} \cdot\left(x_{\mathrm{B}}-x_{\mathrm{p}}\right)$, and additional singularities are needed at the centre of the sphere that cancel the velocity-field correction $u_{2}^{(1)}(x)$ at the sphere surface $x_{\mathrm{B}}$; namely the interface 'reflection' of the potential quadrupole $(\sigma, v)=\left(\frac{1}{2} e_{3}, e_{3}\right)$ and the stresslet $(\boldsymbol{\rho}, \mu)=\left(\frac{5}{2} e_{3}, e_{3}\right)$, which is non-zero at the sphere surface.

Since the detailed form of $u_{2}^{(1)}\left(x_{B}\right)$ is highly complicated, it is not possible to determine singularities at the sphere centre that precisely satisfy the boundary condition $(5 b)$ at all points on the sphere surface. Instead, we choose singularities to cancel only the first few terms of $u_{2}^{(1)}\left(x_{\mathrm{B}}\right)$ at the sphere surface, with $\boldsymbol{u}_{2}^{(1)}\left(x_{\mathrm{B}}\right)$ expanded in powers of $\delta$ for $\delta=a / d \ll 1$. The leading terms of $\boldsymbol{u}_{2}^{(1)}$ near the sphere, for small $\delta$, are in component form,

$$
\begin{gathered}
u_{2}^{(1)}=\delta^{3} \frac{5 x_{1}}{16} \frac{1+2 \lambda}{1+\lambda}+O\left(\delta^{4}\right), \quad v_{2}^{(1)}=\delta^{3} \frac{5 x_{2}}{16} \frac{1+2 \lambda}{1+\lambda}+O\left(\delta^{4}\right), \\
w_{2}^{(1)}=-\delta^{2} \frac{5}{16} \frac{2+3 \lambda}{1+\lambda}-\delta^{3} \frac{5}{8} \frac{1+2 \lambda}{1+\lambda}\left(x_{3}+d\right)+O\left(\delta^{4}\right),
\end{gathered}
$$

where the subscript 2 denotes the velocity components in fluid 2 . It can be seen from $(8 a-c)$ that the presence of the interface will induce a steady streaming flow at $O\left(\delta^{2}\right)$ normal to the interface, and an axisymmetric uniaxial extensional flow at $O\left(\delta^{3}\right)$ with a stagnation point at the sphere centre. The singularities required to cancel this additional velocity field at the sphere surface can be readily evaluated, and the resulting solution for a stationary sphere near a flat interface in the pure straining flow, $\hat{U}_{2}=E \cdot\left(x-x_{p}\right)$, is as follows:

$$
\begin{aligned}
u_{2}\left(x, x_{\mathrm{p}}\right)= & \frac{5}{8}\left[\frac{3}{8} \frac{2+3 \lambda}{1+\lambda} \delta^{2}+\left(\frac{3}{8} \frac{2+3 \lambda}{1+\lambda}\right)^{2} \delta^{3}+O\left(\delta^{4}\right)\right] \boldsymbol{u}_{2, \mathrm{~s}}\left(x, x_{\mathrm{p}} ; \boldsymbol{e}_{3}\right) \quad \text { (Stokeslet) } \\
& +\frac{5}{8}\left[\frac{3}{8} \frac{2+3 \lambda}{1+\lambda} \delta^{2}+\left(\frac{3}{8} \frac{2+3 \lambda}{1+\lambda}\right)^{2} \delta^{3}+O\left(\delta^{4}\right)\right] \boldsymbol{u}_{2, \mathrm{D}}\left(x, x_{\mathrm{p}} ;-\frac{1}{3} e_{3}\right) \quad \text { (potential dipole) } \\
& +\frac{5}{2}\left[1+\frac{5}{16} \frac{1+2 \lambda}{1+\lambda} \delta^{3}+O\left(\delta^{4}\right)\right] \boldsymbol{u}_{2, \mathrm{ss}}\left(x, x_{\mathrm{p}} ; e_{3}, e_{3}\right) \quad \text { (stresslet) } \\
& +\frac{1}{2}\left[1+\frac{5}{16} \frac{1+2 \lambda}{1+\lambda} \delta^{3}+O\left(\delta^{4}\right)\right] \frac{\partial}{\partial x_{3}} \boldsymbol{u}_{2, \mathrm{D}}\left(x, x_{\mathrm{p}} ; \boldsymbol{e}_{3}\right) \quad \text { (potential quadrupole). (9) }
\end{aligned}
$$


Now let us turn to the original problem of calculating the force and torque acting on a stationary sphere that is located at arbitrary point $x_{p}$ in fluid 2 which is undergoing the axisymmetric uniaxial extension flow $\boldsymbol{O}_{2}=\boldsymbol{E} \cdot \boldsymbol{x}$ with origin at the interface (i.e. figure $1 a$ ). As we show in $\$ 2$, the hydrodynamic force and torque exerted in this case can be determined by a superposition of the force and torque for a uniform streaming flow with translational velocity $\boldsymbol{V}_{i}=E \cdot \boldsymbol{x}_{\mathrm{p}}$ and for a uniaxial straining flow $\hat{U}_{i}=E \cdot\left(x-x_{\mathrm{p}}\right)$ with stagnation point at the sphere centre. The force and torque in the latter case can be evaluated directly from the strength of the singularities in the solution (9). The result is

$$
\begin{gathered}
F=-\boldsymbol{K}_{\mathrm{T}} \cdot E \cdot x_{\mathrm{p}}-5 \pi\left[\frac{3}{8} \delta^{2} \frac{2+3 \lambda}{1+\lambda}+\left(\frac{3}{8} \delta \frac{2+3 \lambda}{1+\lambda}\right)^{2} \delta\right] e_{3}+O\left(\delta^{4}\right), \\
T=-\boldsymbol{K}_{\mathrm{C}} \cdot E \cdot \boldsymbol{x}_{\mathrm{p}}+O\left(\delta^{4}\right) .
\end{gathered}
$$

The components of the translation and coupling tensors $\boldsymbol{K}_{\mathrm{T}}$ and $\boldsymbol{K}_{\mathrm{C}}$ were determined up to $O\left(\delta^{2}\right)$ by Lee et al. (1979) for motion of a sphere near a plane fluid-fluid interface. The $O\left(\delta^{3}\right)$ terms in the components of the hydrodynamic tensors, $\boldsymbol{K}_{\mathrm{T}}$ and $\boldsymbol{K}_{\mathrm{C}}$, which are necessary to be compatible with the inclusion of $O\left(\delta^{3}\right)$ terms in (9), can be evaluated by expanding the corresponding wall correction $\boldsymbol{u}_{2}^{(1)}(x)$ up to $O\left(\delta^{3}\right)$ and superimposing the fundamental solutions for singularities in order to cancel the interface reflection at the sphere surface at the same level of approximation $O\left(\delta^{3}\right)$. The resulting non-zero components of the hydrodynamic tensors $\boldsymbol{K}_{\mathrm{T}}, \boldsymbol{K}_{\mathrm{C}}$ are given by

$$
\begin{aligned}
& K_{\mathrm{T}}^{11}=6 \pi\left[1+\sum_{n=1}^{3}\left(\frac{3}{16} \delta \frac{3 \lambda-2}{1+\lambda}\right)^{n}-\frac{1+2 \lambda}{16(1+\lambda)} \delta^{3}\right]+O\left(\delta^{4}\right), \quad K_{\mathrm{T}}^{22}=K_{\mathrm{T}}^{11}, \\
& K_{\mathrm{T}}^{33}=6 \pi\left[1+\sum_{n=1}^{3}\left(\frac{3}{8} \delta \frac{2+3 \lambda}{1+\lambda}\right)^{n}-\frac{1+4 \lambda}{8(1+\lambda)} \delta^{3}\right]+O\left(\delta^{4}\right), \\
& K_{\mathrm{C}}^{12}=\frac{3 \pi}{2} \delta^{2} \frac{1}{1+\lambda}\left[1+\frac{3}{16} \delta \frac{3 \lambda-2}{1+\lambda}\right]+O\left(\delta^{4}\right), \quad \text { and } \quad K_{\mathrm{C}}^{21}=-K_{\mathrm{C}}^{12}
\end{aligned}
$$

Here the terms in the summed series,

$$
\left(\frac{3}{18} \delta \frac{3 \lambda-2}{1+\lambda}\right)^{n} \text { in } K_{\mathrm{T}}^{11} \text { and }\left(\frac{3}{8} \delta \frac{2+3 \lambda}{1+\lambda}\right)^{n} \text { in } K_{\mathrm{T}}^{33}
$$

are the corresponding $n$th reflections to the terms $O(\delta)$ in the first wall corrections $\boldsymbol{u}_{2}^{(1)}(\boldsymbol{x})$, and the summed series continue beyond $O\left(\delta^{3}\right)$. The terms of $O\left(\delta^{3}\right)$,

$$
-\frac{1+2 \lambda}{16(1+\lambda)} \delta^{3} \quad \text { in } K_{\mathrm{T}}^{11} \quad \text { and } \quad-\frac{1+4 \lambda}{8(1+\lambda)} \delta^{3} \quad \text { in } K_{\mathrm{T}}^{33}
$$

result from the correction terms of $O\left(\delta^{3}\right)$ in $\boldsymbol{u}_{2}^{(1)}(\boldsymbol{x})$, which represent a paraboloidal velocity field with origin at the sphere centre and a steady streaming flow, both either parallel or normal to the interface. The term $O\left(\delta^{3}\right)$ in $K_{\mathrm{C}}^{12}$ or $K_{\mathrm{C}}^{21}$ is associated with the reflected simple shearing flow of either the $O(\delta)$ term in $\boldsymbol{u}_{2}^{(1)}(x)$ for translation parallel to the interface or the $O\left(\delta^{2}\right)$ term in $u_{2}^{(1)}(x)$ for rotation with axis of rotation parallel to the interface.

Dukhin \& Rulev (1977) determined the drag force on a small solid sphere located at the axis of symmetry in an axisymmetric uniaxial extensional flow $\hat{O}_{i}=\boldsymbol{E} \cdot \boldsymbol{x}$, near a gas-liquid interface (i.e. $\lambda \rightarrow 0$ ), using the eigensolutions of Laplace's equation in bipolar coordinates. It is a simple matter to calculate the drag force $F_{3}$ 


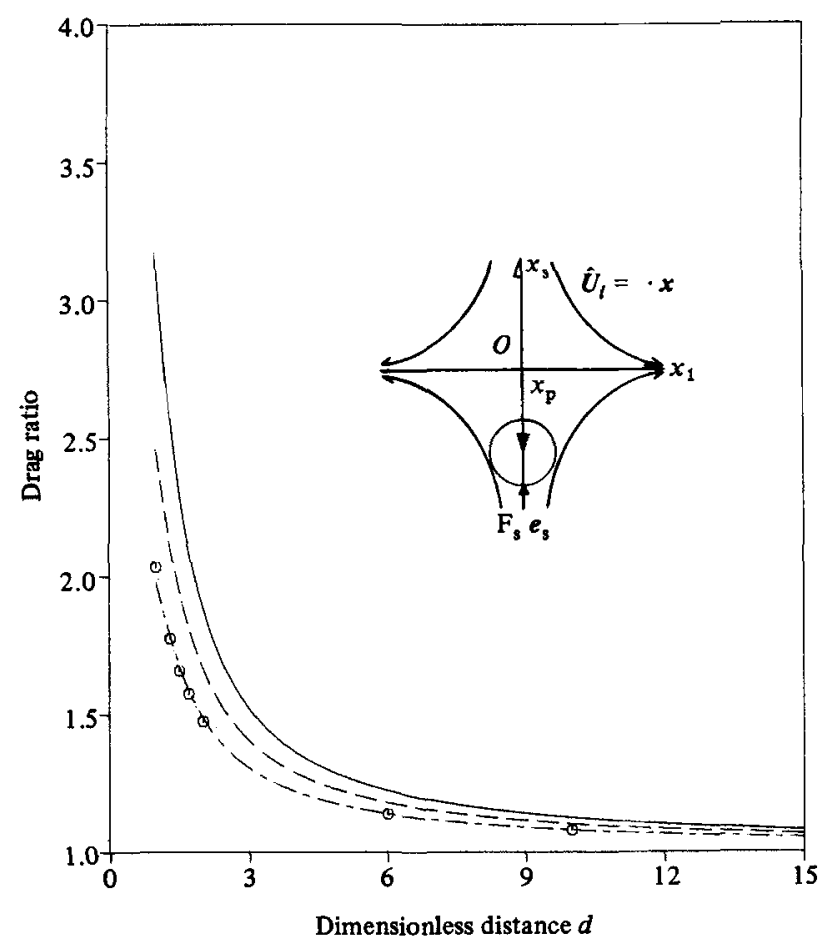

Frgure 2. Drag ratio for axisymmetric extensional flow relative to Stokes drag in an unbounded fluid as a function of the dimensionless distance $d$ between the sphere centre and the interface; $\hat{U}_{2}=\boldsymbol{E} \cdot \boldsymbol{x}:-, \lambda=\infty ;--, 1,----, 0 ; 0$, corresponding exact-solution results $(\lambda=0)$ of Dukhin \& Rulev (1977).

on the sphere from the present asymptotic solution $(10 a)$ with $x_{\mathrm{p}}=(0,0,-d)$. The drag ratio (the drag $F_{3}$ divided by the Stokes drag $12 \pi \mu_{2} a d E$ ) is simply given as

$$
\text { drag ratio }=1+\sum_{n=1}^{3}\left(\frac{3}{8} \delta \frac{2+3 \lambda}{1+\lambda}\right)^{n}-\frac{1+4 \lambda}{8(1+\lambda)} \delta^{3}-\frac{5}{12 d} \sum_{n=1}^{2} \delta\left(\frac{3}{8} \delta \frac{2+3 \lambda}{1+\lambda}\right)^{n}+O\left(\delta^{4}\right)
$$

In figure 2 the drag ratio (12) is plotted as a function of $d$, the distance between the sphere and the stagnation point, for three values of $\lambda=0,1$ and $\infty$. Also shown for comparison is the corresponding exact solution of Dukhin \& Rulev (1977). There is very good agreement between the two solutions, except in the region near $d=1$. As expected, the difference between the two results becomes larger as the sphere approaches the interface owing to the poor convergence of the asymptotic solution (12) in powers of $\delta$. However, a detailed comparison shows that the maximum error in the asymptotic solution (12), compared with the exact solution of Dukhin \& Rulev, is only $2.72 \%$ for $d=1.001$, which is the smallest value considered by Dukhin \& Rulev, while the error for $d>1.5$ becomes less than $0.98 \%$.

\subsection{Simple shear flow}

Let us turn now to the case of a sphere located at an arbitrary point $x_{\mathrm{p}}$ in a simple shear flow $\hat{U}_{i}=\left(\mu_{i} / \mu_{2}\right) \boldsymbol{\Gamma} \cdot \boldsymbol{x}$, parallel to the interface as shown in figure $1(d)$ (the case in which $O_{i}=C \neq 0$ at the interface can be treated by superimposing a uniform streaming flow past a sphere, $\hat{U}_{i}=C$, with the simple shear flow $\left.\hat{U}_{i}=\left(\mu_{i} / \mu_{2}\right) \Gamma \cdot x\right)$. Again, the problem can be decomposed into a simple translation of the fluid system 
including the interface with velocity $\boldsymbol{O}_{i}=\mathbf{\Gamma} \cdot \boldsymbol{x}_{\mathrm{p}}$ past the stationary sphere (i.e. figure $1 e)$ together with a linear shear flow $\sigma_{i}=\left(\mu_{i} / \mu_{2}\right) \Gamma \cdot x-\Gamma \cdot x_{p}$ with $\sigma_{2}=0$ at the sphere centre (i.e. figure $1 f$ ). In view of the linearity of the problem and the symmetry of the sphere-interface geometry, we need only solve the case of $\sigma_{i}=$ $\left(\mu_{i} / \mu_{2}\right) \Gamma_{13} \cdot x_{3} e_{1}$, corresponding to

$$
\boldsymbol{L}=\boldsymbol{\Gamma}=\left[\begin{array}{ccc}
0 & 0 & \Gamma_{13} \\
0 & 0 & 0 \\
0 & 0 & 0
\end{array}\right] \text {, shear rate tensor. }
$$

In order to analyse the velocity field for a sphere in the undisturbed flow $\sigma_{i}=\Gamma_{13}\left[\left(\mu_{i} / \mu_{2}\right) x_{3}+d\right] e_{1}$, which vanishes at the sphere centre, we follow the procedure of $\$ 3.1$ and solve the equivalent problem in which a velocity field $u_{2}(x)$ is viewed as being generated in a quiescent fluid by a non-zero velocity distribution

$$
u_{2}\left(x_{\mathrm{B}}\right)=-\Gamma_{13}\left(\left(x_{\mathrm{B}}\right)_{3}+d\right) e_{1}
$$

at the surface of the sphere. As in the preceding analysis, we use the method of reflections, with the solution in an unbounded fluid taken from the work of Chwang $\& W u(1975)$, who showed that the condition (13) was satisfied by superposition of a stresslet, a rotlet and a potential quadrupole at the centre of the sphere, i.e.

$$
\begin{aligned}
\text { stresslet } & (\rho, \mu)=\left(-\frac{5}{6} \Gamma_{13} e_{1}, e_{3}\right), \\
\text { rotlet } & \gamma=-\frac{1}{2} \Gamma_{13} e_{2} \\
\text { potential quadrupole } & (\sigma, v)=\left(-\frac{1}{6} \Gamma_{13} e_{3}, e_{1}\right)
\end{aligned}
$$

As in the preceding example, the first correction for the presence of the interface in the reflections expansion can now be obtained easily from Chwang \& Wu's solution by simply replacing the fundamental solutions $u_{\mathrm{R}}, u_{\mathrm{D}}$ and $\boldsymbol{u}_{\mathrm{SS}}$ (which pertain to an unbounded fluid) with the corresponding fundamental solutions $u_{2, \mathrm{R}}, \boldsymbol{u}_{2, \mathrm{D}}$ and $\boldsymbol{u}_{2, \mathrm{sS}}$ that satisfy boundary conditions on the flat interface (and are generated using the procedure of Lee et al. 1979). The result is the first two terms in the reflections expansion, i.e. $\left(u_{2}^{(0)}+u_{2}^{(1)}, p_{2}^{(0)}+p_{2}^{(1)}\right)$. Subtracting the zeroth-order (Chwang \& Wu 1975 ) solution, we get

$$
u_{2}^{(1)}(x)=-\Gamma_{13}\left[\frac{5}{8} u_{2, \mathrm{SS}}\left(x, x_{\mathrm{p}} ; e_{1}, e_{3}\right)+\frac{1}{2} u_{2, \mathrm{R}}\left(x, x_{\mathrm{p}} ; e_{2}\right)+\frac{1}{6} \frac{\partial}{\partial x_{3}} u_{2, \mathrm{D}}\left(x, x_{\mathrm{p}} ; e_{1}\right)\right]-u_{2}^{(0)}(x)
$$

Although the combined solution $\left(\boldsymbol{u}_{2}^{(0)}+\boldsymbol{u}_{2}^{(1)}, p_{2}^{(0)}+p_{2}^{(1)}\right)$ satisfies the boundary conditions at the interface, the boundary condition (13) on the sphere is not satisfied, because the 'reflected flow field' $u_{2}^{(1)}$ is non-zero at the sphere surface. Following $\$ 3.1$, we examine the leading terms of this reflected field at the sphere surface as a power series in $\delta$ :

and

$$
u_{2}^{(1)}=\delta^{2} \frac{5 \lambda-2}{16(1+\lambda)} \Gamma_{13}+\delta^{3} \frac{1}{8} \frac{3 \lambda-1}{(1+\lambda)} \Gamma_{13}\left(x_{3}+d\right)+O\left(\delta^{4}\right), \quad v_{2}^{(1)}=O\left(\delta^{4}\right)
$$

$$
w_{2}^{(1)}=\delta^{3} \frac{1}{8} \frac{2+3 \lambda}{(1+\lambda)} \Gamma_{13} x_{1}+O\left(\delta^{4}\right)
$$

Thus, insofar as $(15 a-c)$ are concerned, the presence of the interface is equivalent to an induced steady simple shear flow at $O\left(\delta^{8}\right)$ either normal or parallel to the interface and a steady streaming motion at $O\left(\delta^{2}\right)$ parallel to the flow field (i.e. $e_{1}$ direction).

In order to satisfy the condition (13), additional singularities are required at the sphere centre. These can be determined following the procedures of Lee et al. (1979), 
as well as those of $\S 3.1$. The resulting solution, expressed in terms of the fundamental solutions for a Stokeslet, potential dipole and higher-order singularities near an interface (Lee et al. 1979), is

$$
\begin{aligned}
u_{2}\left(x, x_{\mathrm{p}}\right)= & u_{2, \mathrm{~s}}\left(x, x_{\mathrm{p}} ; e_{1}\right)\left[\frac{-3}{64} \Gamma_{13} \frac{5 \lambda-2}{\lambda+1} \delta^{2}\left(1-\frac{3}{16} \frac{2-3 \lambda}{1+\lambda} \delta\right)+O\left(\delta^{4}\right)\right] \quad \text { (Stokeslet) } \\
& +u_{2, \mathrm{D}}\left(x, x_{\mathrm{p}} ;-\frac{1}{3} e_{1}\right)\left[\frac{-3 \Gamma_{13}}{64} \frac{5 \lambda-2}{1+\lambda} \delta^{2}\left(1-\frac{3}{16} \frac{2-3 \lambda}{1+\lambda} \delta\right)+O\left(\delta^{4}\right)\right] \quad \text { (potential dipole) } \\
& +u_{2, \mathrm{ss}}\left(x, x_{\mathrm{p}} ; e_{1} e_{3}\right) \frac{5 \Gamma_{13}}{6}\left[-1-\frac{1}{8} \frac{1+6 \lambda}{1+\lambda} \delta^{3}+O\left(\delta^{4}\right)\right] \quad \text { (stresslet) } \\
& +u_{2, \mathrm{R}}\left(x, x_{\mathrm{p}} ; e_{2}\right) \frac{\Gamma_{13}}{2}\left[-1+\frac{3}{8} \frac{1}{1+\lambda} \delta^{3}+O\left(\delta^{4}\right)\right] \quad(\text { rotlet) } \\
& +\frac{\partial}{\partial x_{3}} u_{2, \mathrm{D}}\left(x, x_{\mathrm{p}} ; e_{1}\right) \frac{\Gamma_{13}}{6}\left[-1-\frac{1}{8} \frac{3 \lambda-1}{1+\lambda} \delta^{3}\right]-\frac{\partial}{\partial x_{1}} u_{2, \mathrm{D}}\left(x, x_{\mathrm{p}} ; e_{3}\right) \frac{\Gamma_{13}}{48} \frac{2+3 \lambda}{1+\lambda} \delta^{3} \\
& +O\left(\delta^{4}\right) \quad \text { (potential quadrupole). }
\end{aligned}
$$

From this solution and $(3 a, b)$, we can easily determine the hydrodynamic force and torque exerted on a sphere located at an arbitrary point $x_{p}$, in the simple shear flow $\hat{U}_{i}=\left(\mu_{i} / \mu_{2}\right) \Gamma \cdot x$, with $\hat{U}_{i}=0$ at the interface. This result is

$$
F=-K_{\mathrm{T}} \cdot \boldsymbol{\Gamma} \cdot \boldsymbol{x}_{\mathrm{p}}+K_{\mathrm{SF}} \cdot \xi_{\mathrm{s}}, \quad T=-K_{\mathrm{c}} \cdot \boldsymbol{\Gamma} \cdot \boldsymbol{x}_{\mathrm{p}}+\boldsymbol{K}_{\mathrm{ST}} \cdot \boldsymbol{\xi}_{\mathrm{s}},
$$

in which $\xi_{\mathrm{s}}$ is defined by $\xi_{\mathrm{s}}=\left(\Gamma_{13}, \Gamma_{23}, 0\right)$, and the non-zero components of the hydrodynamic tensors $\boldsymbol{K}_{\mathrm{SF}}$ and $\boldsymbol{K}_{\mathrm{ST}}$ are given by

$$
\begin{gathered}
K_{\mathrm{SF}}^{11}=\frac{3 \pi}{8} \frac{5 \lambda-2}{1+\lambda} \delta^{2}\left[1-\frac{3}{16} \frac{2-3 \lambda}{1+\lambda} \delta\right]+O\left(\delta^{4}\right), \quad K_{\mathrm{SF}}^{22}=K_{\mathrm{SF}}^{11}, \\
K_{\mathrm{ST}}^{22}=-4 \pi\left[1-\frac{1}{8} \frac{3}{1+\lambda} \delta^{3}\right]+O\left(\delta^{4}\right), \quad K_{\mathrm{ST}}^{21}=-K_{\mathrm{ST}}^{12} .
\end{gathered}
$$

The drag ratio (the drag divided by the Stokes drag $-6 \pi \mu_{2} \Gamma_{13} d a$ ) is simply given as

$$
\begin{aligned}
\text { drag ratio }=1+\sum_{n=1}^{3}(-1)^{n}\left(\frac{3}{16} \delta \frac{2-3 \lambda}{1+\lambda}\right)^{n} & -\frac{1+2 \lambda}{16(1+\lambda)} \delta^{3} \\
& +\frac{1}{16 d} \delta^{2} \frac{2-5 \lambda}{1+\lambda}\left[1-\frac{3}{16} \delta \frac{2-3 \lambda}{1+\lambda}\right]+O\left(\delta^{4}\right),
\end{aligned}
$$

where we have again adopted $\xi_{\mathrm{s}}=\left(\Gamma_{13}, 0,0\right)$ with no loss of generality.

For a simple shear flow parallel to a rigid plane boundary, Goren \& O'Neill (1971) calculated the hydrodynamic force and torque on a sphere, using the eigensolutions of Laplace's equation in bipolar coordinates developed by Jeffery (1912). In figure 3 the drag ratio (19) is plotted as a function of $d$, the distance between the sphere and the interface, for three values of $\lambda=0,1$ and $\infty$. Also shown for comparison are the corresponding drag ratios determined by Goren \& O'Neill. As mentioned previously, we presume $\delta \ll 1$ in the derivation of (19). Thus for $\delta \ll 1$ (i.e. $d \gg 1$ ) the asymptotic solution (19) coincides almost exactly with Goren \& O'Neill's result, which is the exact solution for the simple shear flow parallel to a solid wall. Even for $d \approx 1.5$, the approximate solution shows reasonably good agreement with the exact solution. Indeed, the relative error is within $2.6 \%$ for $d>1.5$. Wakiya (1957) considered the case of a sphere in a linear shear flow between two rigid parallel flat planes (i.e. $\lambda \rightarrow \infty$ ), in which one plane is held stationary and the other is moved parallel to itself under 


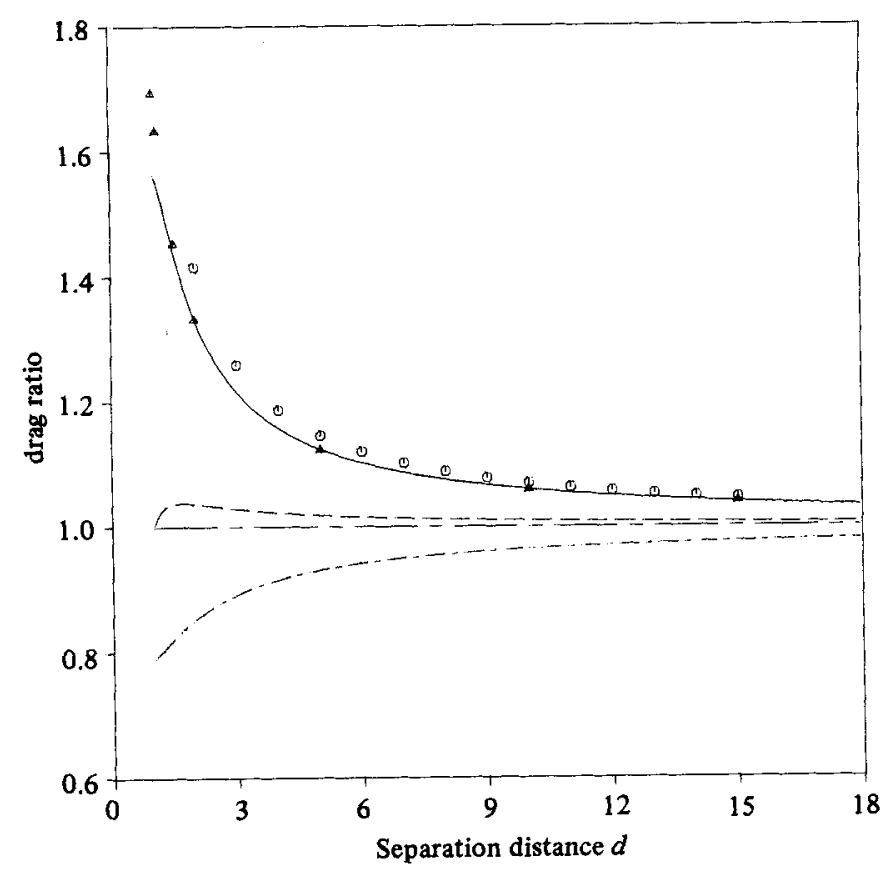

Figure 3. Drag ratio relative to the drag in an unbounded fluid as a function of the dimensionless distance $d$ between the sphere centre and the interface; $\theta_{2}=\Gamma_{13} x_{3} e_{1}:-, \lambda=\infty ;---, 1$; $\ldots$ (1957) for two parallel plates.

the assumption that the motion of the sphere is parallel to the walls. Wakiya determined the drag and torque on the sphere located at a distance $d$ from the stationary plate and $3 d$ away from the moving plate, using the general method developed by Faxén (1921). The drag ratios calculated asymptotically by Wakiya for the limit $\delta \ll 1$ are also shown in figure 3. As might be expected, Wakiya's results converge to the asymptotic solution, (19) with $\lambda \rightarrow \infty$, as the distance $d$ is increased, since the effect of the moving plate becomes negligible compared with the effect of the stationary plate with increase in the distance.

The hydrodynamic torque on a sphere in the flow $\hat{O}_{i}=\left(\mu_{i} / \mu_{2}\right) \mathbf{\Gamma} \cdot \boldsymbol{x}$ can be evaluated from $(17 b)$, and is equal to

$$
T=4 \pi \Gamma_{13}\left[1+\frac{3}{8} \delta \frac{1}{1+\lambda}\left(1-\frac{3}{16} \delta \frac{2-3 \lambda}{1+\lambda}-\delta^{2}\right)\right] e_{2}+O\left(\delta^{4}\right) .
$$

This is the negative of the torque that is required to keep the sphere from rotating. It can be compared directly with the corresponding results from Goren \& O'Neill's exact solution for a single rigid wall and from Wakiya's asymptotic solution for two parallel plates with the same sphere location (i.e. $d$ away from one plate and $3 d$ away from the other). There is good agreement between the asymptotic solution (20) and the exact solution of Goren \& O'Neill (1971) in the region of $\delta \ll 1$, though it can be noted from (20) that, when $\lambda \rightarrow \infty$, the interface contribution to the torque $T$ is zero through $O\left(\delta^{4}\right)$. Although the discrepancy between the two solutions becomes larger as $\delta \rightarrow 1$, it still remains relatively small (e.g. the relative error at $d=1.01$ is only $5.84 \%$ and the error is within $3 \%$ for $d>1.5$ ). As expected, Wakiya's solution also approaches the asymptotic solution for $\lambda \rightarrow \infty$ as the distance $d$ is increased. However, 
for the two-parallel-plate case the torque is increased in magnitude by the presence of plane boundaries in contradiction to the single-wall case.

In this section we have determined the solutions of Stokes' equations for a sphere at rest at an arbitrary point either in a pure straining flow or in a simple shear flow near a fluid-fluid interface with an arbitrary viscosity ratio. We shall turn shortly to the application of these solutions for trajectory calculations. First, however, we consider corresponding solutions for a rod-like slender body.

\section{Solutions for a slender body}

Let us turn now to the case of a rod-like slender body whose centre is located at an arbitrary point $x_{p}$ near an interface in the presence of a linear undisturbed flow field (pure straining or simple shear), $\sigma_{i}=\boldsymbol{L}_{i} \cdot \boldsymbol{x}$ with origin at the interface. The slender body is assumed to be at rest and completely immersed in fluid 2 with an arbitrary orientation which can be expressed in terms of Euler angles $\theta$ and $\phi$ relative to the interface. For present purposes, we define $\theta$ as the oblique angle between the body axis and the interface, while $\phi$ is a subtended angle between the $\left(x_{1}, x_{3}\right)$-plane and the plane defined by the body axis and vector $e_{3}$ normal to the interface (cf. figure 4). At the outset, we assume that the body is oriented with arbitrary oblique angle $\theta$, but that $\phi=0^{\circ}$. Thus, the projection of the body axis onto the interface exactly coincides with the $x_{1}$ axis. The solution for an arbitrary $\phi$-orientation can be simply obtained from the case of $\phi=0^{\circ}$, by use of an orthogonal rotation tensor $\boldsymbol{Q}$ defined by

$$
\boldsymbol{Q}=\left[\begin{array}{ccc}
\cos \phi & \sin \phi & 0 \\
-\sin \phi & \cos \phi & 0 \\
0 & 0 & 1
\end{array}\right]
$$

\subsection{Pure straining flows}

Now let us turn to the case of a slender body held with its centre fixed at an arbitrary point $x_{\mathrm{p}}$ in a uniaxial axisymmetric extension flow $\hat{U}_{i}=\boldsymbol{E} \cdot \boldsymbol{x}$ with stagnation point at the interface. The problem can be treated, as in the case of a sphere, by decomposing the undisturbed flow into a simple translation $\sigma_{i}=E \cdot x_{p}$ past the slender body and a linear flow $\sigma_{i}=E \cdot\left(x-x_{\mathrm{p}}\right)$ with stagnation point at $x_{\mathrm{p}}$. The simple translation problem was treated in Part 1 of this series. Here we solve the problem with undisturbed flow $\hat{U}_{i}=\boldsymbol{E} \cdot\left(\boldsymbol{x}-\boldsymbol{x}_{\mathrm{p}}\right)$. For this purpose, it is convenient to consider the equivalent problem in which the body generates a velocity field $u_{2}\left(x_{B}\right)=-E \cdot\left(x_{B}-x_{p}\right)$ at the body surface, i.e. we solve

$$
-E \cdot\left(x_{\mathrm{B}}-x_{\mathrm{p}}\right)=\int_{-1}^{1}\left[\alpha\left(x_{\mathrm{s}}\right)-\frac{1}{2} \beta\left(x_{\mathrm{s}}\right) \nabla^{2}\right] \cdot \psi\left(x_{\mathrm{B}}, x_{\mathrm{s}}\right) \mathrm{d} \zeta,
$$

in which $\psi\left(x_{\mathrm{B}}, \boldsymbol{x}_{\mathrm{s}}\right)$ denotes the Cartesian tensorial Green function for a unit point force located at $x_{\mathrm{s}}$ (cf. Yang \& Leal 1983).

The integral (21) cannot be solved exactly (except in a numerical sense), but can be solved approximately by means of an asymptotic expansion for small $1 / \kappa$ and $R_{0} / d$, where $R_{0}$ is the maximum radius of the body cross-section. By expanding (21) to $O\left(1 / \kappa, R_{0} / d\right)$ with $\alpha(\zeta)=\left(\alpha_{1}(\zeta), 0, \alpha_{3}(\zeta)\right)$ and $\beta(\zeta)=\left(\beta_{1}(\zeta), 0, \beta_{3}(\zeta)\right)$, it can be shown that the potential dipole strength $\beta(x)$ and the Stokeslet strength $\alpha(x)$ must be related according to

$$
\boldsymbol{\beta}(x)=-\frac{1}{2} r_{0}^{2}(x) \boldsymbol{\alpha}(x)
$$

in order to satisfy the no-slip condition $(5 b)$ at the body surface to $O\left(\epsilon^{2}\right)$. 


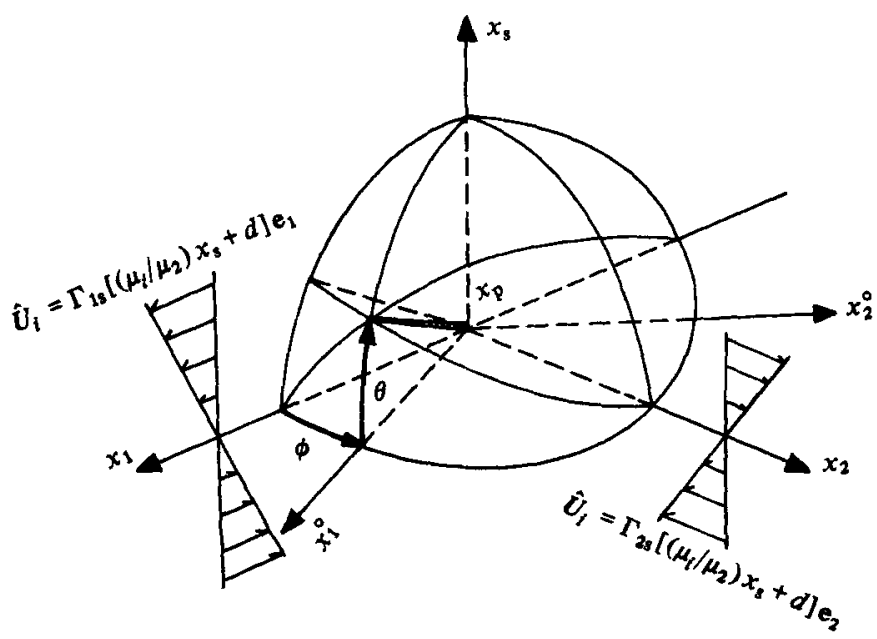

Figure 4. Orientation of a slender body in a simple shearing flow with the origin at the body centre. The $\left(x_{1}, x_{2}\right)$-plane is parallel to the interface.

The simultaneous equations, which are obtained from (21) by substituting for $\boldsymbol{\beta}(x)$ according to (22), can be solved by expansion of $\alpha(x)$ in powers of $\epsilon$ for $\epsilon \ll 1$. The use of an expansion in $\epsilon$ to obtain an approximate solution of this type has been widely reported (cf. Batchelor 1970) for motion of a slender body in a single unbounded fluid, and was used by us in Part 1 for simple translation and rotation near an interface. The resulting line distribution of Stokeslets, in component form, is

$$
\alpha_{1}(x)=-\frac{\left(1+\sin ^{2} \theta\right) \cos \theta}{4} x\left[\epsilon-\frac{\epsilon^{2}}{2}\left(2 S(x)+\frac{\sin ^{2} \theta-3}{1+\sin ^{2} \theta}+U(x ; \lambda, \theta, d)\right)\right]
$$

and

$$
-\frac{1}{2} \sin ^{2} \theta \cos \theta x\left[\epsilon-\frac{1}{2} \epsilon^{2}(2 S(x)+1+X(x ; \lambda, \theta, d))\right]
$$

$$
\alpha_{3}(x)=\frac{1}{4} \sin \theta \cos ^{2} \theta x\left[\epsilon-\frac{1}{2} \epsilon^{2}(2 S(x)+1+V(x ; \lambda, \theta, d))\right]
$$

$$
+\frac{\left(1+\cos ^{2} \theta\right) \sin \theta}{2} x\left[\epsilon-\frac{\epsilon^{2}}{2}\left(2 S(x)+\frac{\cos ^{2} \theta-3}{1+\cos ^{2} \theta}+Y(x ; \lambda, \theta, d)\right)\right],
$$

where

$$
S(x)=\ln \left[\frac{\left(1-(x / l)^{2}\right)^{\frac{1}{2}}}{r_{0}(x) / R_{0}}\right]
$$

and $r_{0}(x)$ is the radius of the body cross-section, which is a function of distance $x$ along the body centreline and has a maximum value of $R_{0}$. Here $U(x ; \lambda, \theta, d), X(x ; \lambda, \theta, d)$, $V(x ; \lambda, \theta, d)$ and $Y(x ; \lambda, \theta, d)$ represent the effects of the interface on the slender body and vanish as $d \rightarrow \infty$ (see Appendix $\dagger$ for specific formulae of these functions).

The net force and torque exerted on a slender body located at the stagnation point in the undisturbed flow field $\hat{O}_{i}=\boldsymbol{E} \cdot\left(\boldsymbol{x}-\boldsymbol{x}_{\mathrm{p}}\right)$ can be evaluated simply from the Stokeslet distribution and expressed in the following form:

$$
F=-K_{\mathrm{PF}} \cdot \xi_{\mathrm{p}}, \quad T=-\boldsymbol{K}_{\mathrm{PT}} \cdot \xi_{\mathrm{p}}
$$

$\dagger$ The Appendix is not reproduced here. A copy may be obtained or request from either the authors or the Editor of the Journal. 
where the vector $\xi_{\mathrm{p}}=(1,1,-2)$, and the non-zero components of the hydrodynamic resistance tensor $\boldsymbol{K}_{\mathrm{PF}}$ are

$$
\begin{aligned}
& K_{\mathrm{PF}}^{11}=-\epsilon^{2} \pi\left(1+\sin ^{2} \theta\right) \cos \theta \int_{-1}^{1} x U(x ; \lambda, \theta, d) \mathrm{d} x+O\left(\epsilon^{3}\right), \\
& K_{\mathrm{PF}}^{31}=\epsilon^{2} \pi \sin \theta \cos ^{2} \theta \int_{-1}^{1} x V(x ; \lambda, \theta, d) \mathrm{d} x+O\left(\epsilon^{3}\right), \\
& K_{\mathrm{PF}}^{13}=\epsilon^{2} \pi \cos \theta \sin ^{2} \theta \int_{-1}^{1} x X(x ; \lambda, \theta, d) \mathrm{d} x+O\left(\epsilon^{3}\right), \\
& K_{\mathrm{PF}}^{33}=-\epsilon^{2} \pi\left(1+\cos ^{2} \theta\right) \sin \theta \int_{-1}^{1} x Y(x ; \lambda, \theta, d) \mathrm{d} x+O\left(\epsilon^{3}\right) .
\end{aligned}
$$

The tensor $\boldsymbol{K}_{\mathrm{PT}}$ has the following non-zero components:

$$
\begin{array}{r}
K_{\mathrm{PT}}^{21}=\epsilon \frac{8}{3} \pi \sin \theta \cos \theta\left[1-\epsilon\left(\ln 2-\frac{11}{6}+\frac{3}{8} \int_{-1}^{1}\left[\left(1+\sin ^{2} \theta\right) U(x ; \lambda, \theta, d)\right.\right.\right. \\
\left.\left.\left.+\cos ^{2} \theta V(x ; \lambda, \theta, d)\right] x^{2} \mathrm{~d} x\right)\right]+O\left(\epsilon^{3}\right), \\
K_{\mathrm{PT}}^{23}=-\epsilon \frac{8}{3} \pi \sin \theta \cos \theta\left[1-\epsilon\left(\ln 2-\frac{11}{6}+\frac{3}{8} \int_{-1}^{1}\left[\left(1+\cos ^{2} \theta\right) Y(x ; \lambda, \theta, d)\right.\right.\right. \\
\left.\left.\left.+\sin ^{2} \theta X(x ; \lambda, \theta, d)\right] x^{2} \mathrm{~d} x\right)\right]+O\left(\epsilon^{3}\right) .
\end{array}
$$

In figures 5 and 6 the force components $F_{1}$ and $F_{3}$ of $(24 a)$ are plotted as a function of the orientation angle $\theta$ for $\epsilon=0.1883$, which corresponds to $\kappa=100$, and $d=1.01$ and 2.0. In each case we consider three values of $\lambda=0,1$ and $\infty$. In an unbounded single fluid the net force on a particle at the stagnation point of a linear straining flow would be zero. Obviously, in the case of a sphere, this parallel force component is zero owing to the symmetry of the sphere. The existence of a non-zero force component, $F_{3}$, as shown in figure 6 , was also found for the sphere. The force component $F_{3}$ is always oriented away from the interface, and the magnitude is increased as the viscosity ratio $\lambda$ becomes larger, which is exactly the same as for the sphere (compare (10a) and figure 6 ). Thus a positive external force $-F_{3}$ would have to be applied to the body to keep it from translating away from the stagnation point $x_{\mathrm{p}}$ of the flow regardless of the particle orientation and position, or the viscosity ratio of the two fluids. It should be understood that, in this flow field $O_{i}=E \cdot\left(x-x_{p}\right)$ of figure $1 c$, the interface translates with velocity $-2 d e_{3}$ toward the stagnation point $\boldsymbol{x}_{\mathrm{p}}$ at which the body centre is held fixed. This 'interface motion' can be viewed as the source of both $F_{1}$ and $F_{3}$.

The hydrodynamic torque, $T_{2}$ of $(24 b)$, is non-zero even in an unbounded single fluid, but is significantly modified in the presence of an interface. The torque $T_{2}$ is plotted in figure 7 as a function of the orientation angle $\theta$, for $d=1.01$ and three values of $\lambda=0,1$ and $\infty$. The corresponding result in an unbounded single fluid is almost identical with the case $\lambda=0$. It is evident, since $T_{2} \neq 0$, that a freely suspended slender body (i.e. one with $T=0$ ) would rotate in a direction that depends on $\lambda$, and on the orientation and position of the body relative to the interface (i.e. $\theta$ and $d$ ). For $\lambda=0$ and 1 there exist two possible equilibrium orientations, at which $T_{2}=0$, and this is also true in a single unbounded fluid. However, only one of these, $\theta=0^{\circ}$, is stable, while the other, $\theta=90^{\circ}$, is unstable. When $\theta=0^{\circ}$ the particle axis is parallel to the interface. On the other hand, for $\lambda=\infty$ there exist two unstable equilibrium 


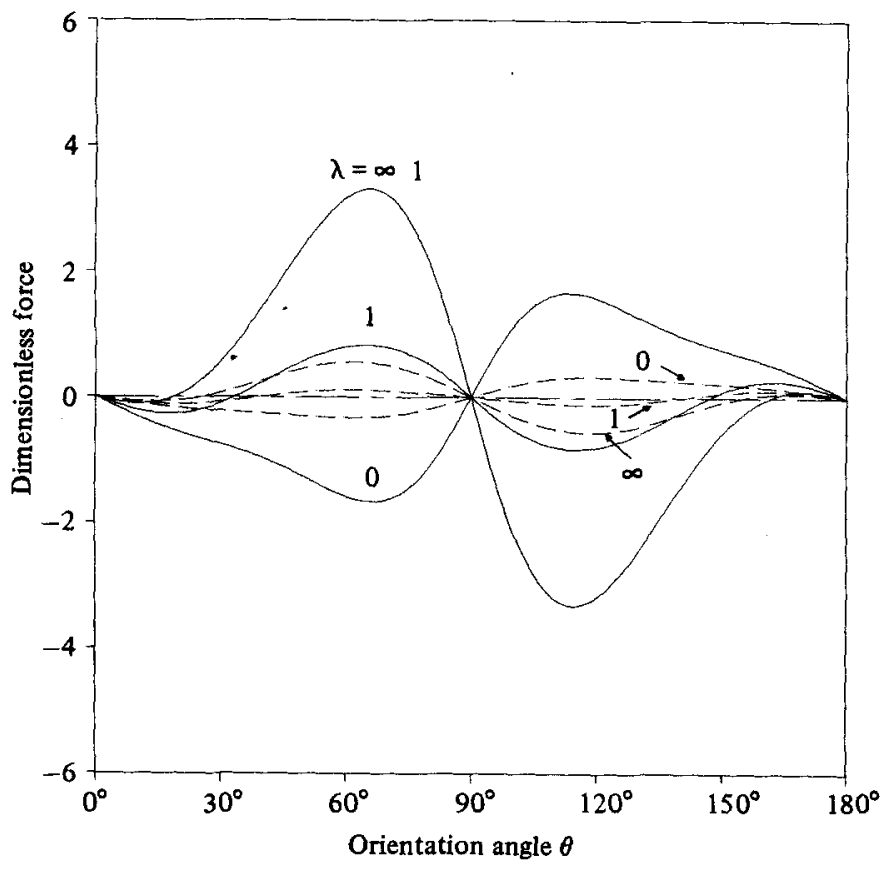

Figure 5. Dimensionless force, $F_{1} / \epsilon^{2} \mu_{2} E l^{2}$ of $(24 a)$ as a function of the orientation angle $\theta$; $\hat{U}_{2}=E \cdot\left(x-x_{p}\right), \epsilon=0.1887, S(x)=\frac{1}{2} \ln \left[1-(x / l)^{2}\right]:-, d=1.01 ;-\square-, 2.0 ;$ unbounded-fluid case.

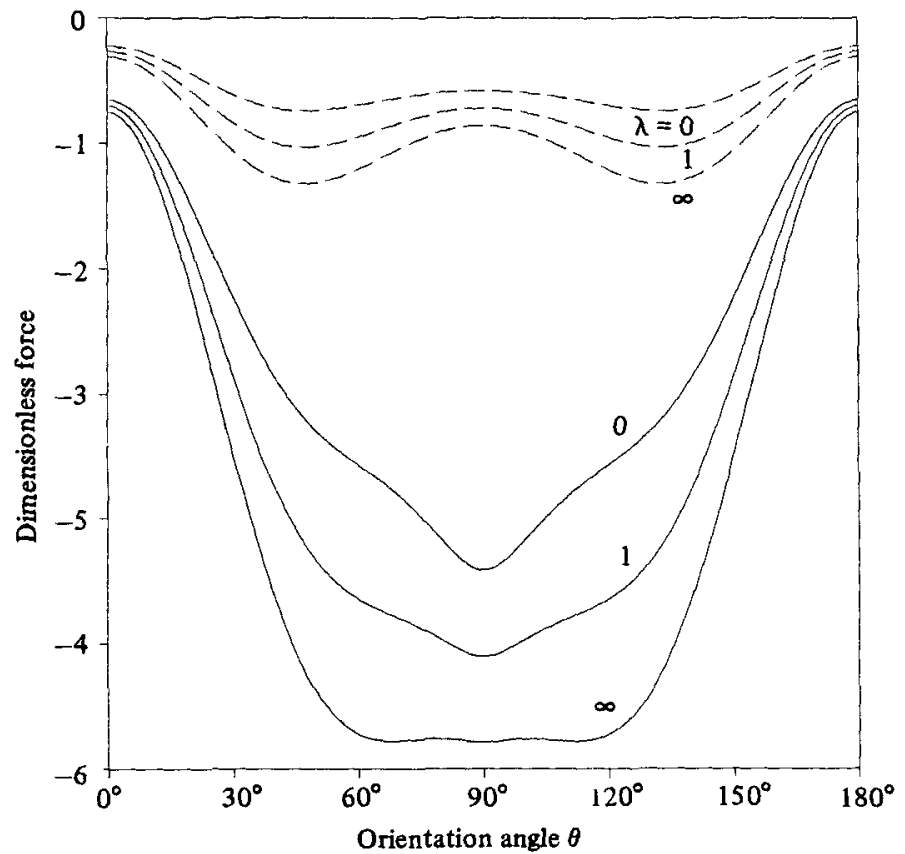

FiguRE 6. Dimensionless force $F_{3} / \epsilon^{2} \mu_{2} E l^{2}$ of $(24 a)$ as a function of the orientation angle $\theta$;

$$
G_{2}=E \cdot\left(x-x_{p}\right), \epsilon=0.1887, S(x)=\frac{1}{2} \ln \left[1-(x / l)^{2}\right]:-, d=1.01,--, 2.0 \text {. }
$$




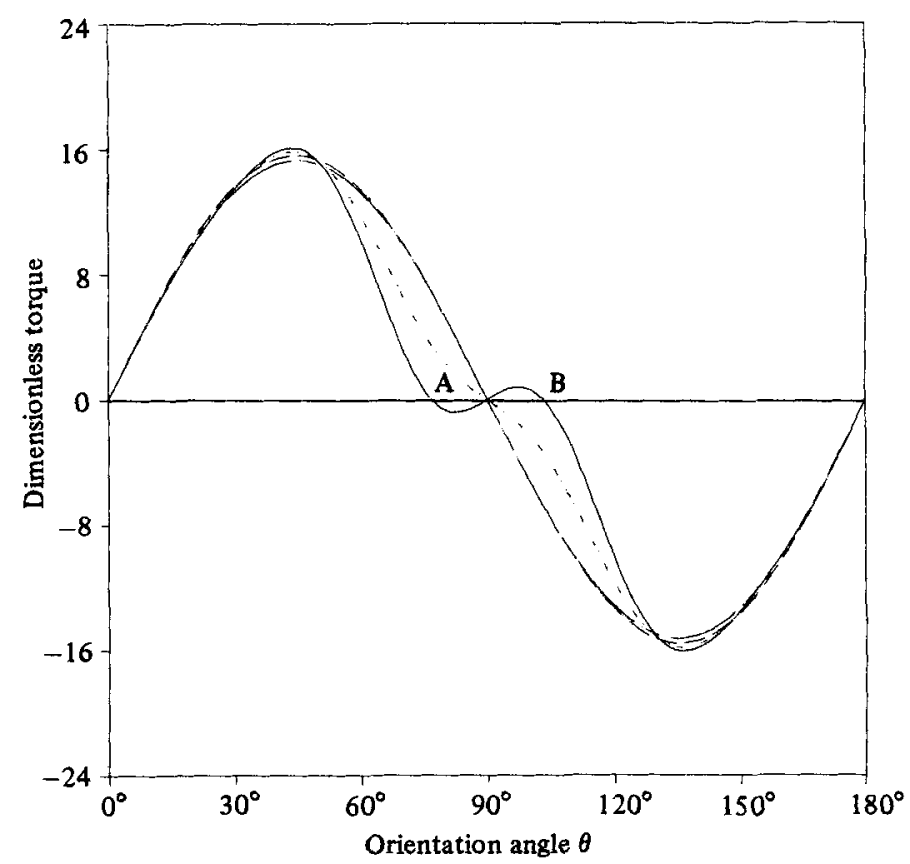

Figure 7. Dimensionless torque $T_{2} / \epsilon \mu_{2} E l^{3}$ of $(24 b)$ as a function of the orientation angle $\theta$; $\hat{O}_{2}=E \cdot\left(x-x_{\mathrm{p}}\right), \epsilon=0.1887, S(x)=\frac{1}{2} \ln \left[1-(x / l)^{2}\right], d=1.01:-, \lambda=\infty ;-\cdot-\cdots, 1 ;-\cdots, 0$, unbounded-fluid case.

orientations corresponding to points $A$ and $B$ in figure 7 , and two stable equilibrium orientations with the particle axis either parallel or perpendicular to the solid wall. The equilibrium orientation that would ultimately be attained in this case by a freely suspended body depends on its initial orientation. It should be noted that the qualitative features evident in figure 7 for $\lambda=\infty$ (i.e. the existence of two stable and two unstable equilibrium orientations) will occur whenever the viscosity ratio $\lambda$ is larger than a critical value (e.g. $\lambda_{\text {cr }}=3.273$ for $d=1.01$ ), for which the two unstable equilibrium orientations overlap exactly at the perpendicular orientation (i.e. the unique unstable equilibrium $\left.\theta=90^{\circ}\right)$. A detailed examination of $(24 b)$ shows that the two unstable equilibrium angles, for a given viscosity ratio, are also shifted to $\theta=90^{\circ}$ as the separation distance $d$ is increased. For example, for $\lambda \rightarrow \infty$, the two equilibrium angles $\theta_{\mathrm{e}}$ are $90^{\circ} \pm 13.28^{\circ}$ for $d=1.01$ (which is the case illustrated in figure 7 ), but become equal to $90^{\circ} \pm 10^{\circ}$ for $d=1.216$, and eventually become coincident at $\theta=90^{\circ}$ for a critical distance $d_{\mathrm{cr}}=1.409$, beyond which there exist only the two distinct equilibrium orientations, $\theta=0^{\circ}$ (stable) and $\theta=90^{\circ}$ (unstable), independent of the viscosity ratio $\lambda$ of the two fluids for a given $\epsilon=0.1887$.

In figure 8 the critical viscosity ratio $\lambda_{\mathrm{cr}}$ is plotted as a function of the separation distance for three values of the aspect ratio $\kappa=20,50$ and 100 , which correspond to $\epsilon=0.2711,0.2171$ and 0.1887 respectively. It can be seen that the critical viscosity ratio is increased, for any given distance $d\left(<d_{\mathrm{cr}}\right)$, as the body becomes more slender, while the critical distance $d_{\mathrm{cr}}$ is decreased (i.e. $d_{\mathrm{cr}}=1.876$ for $\kappa=20,1.580$ for $\kappa=50$, and 1.409 for $\kappa=100$ ). Thus, for a given aspect ratio (or $\epsilon$ ), the condition for existence of the two stable $\left(\theta=0^{\circ}\right.$ and $\left.90^{\circ}\right)$ and two unstable equilibrium orientations is $\lambda>\lambda_{\mathrm{cr}}$ for a distance $d<d_{\mathrm{cr}}$. The implication of these somewhat complicated results for trajectories of a slender body in an extensional flow will be considered later. 


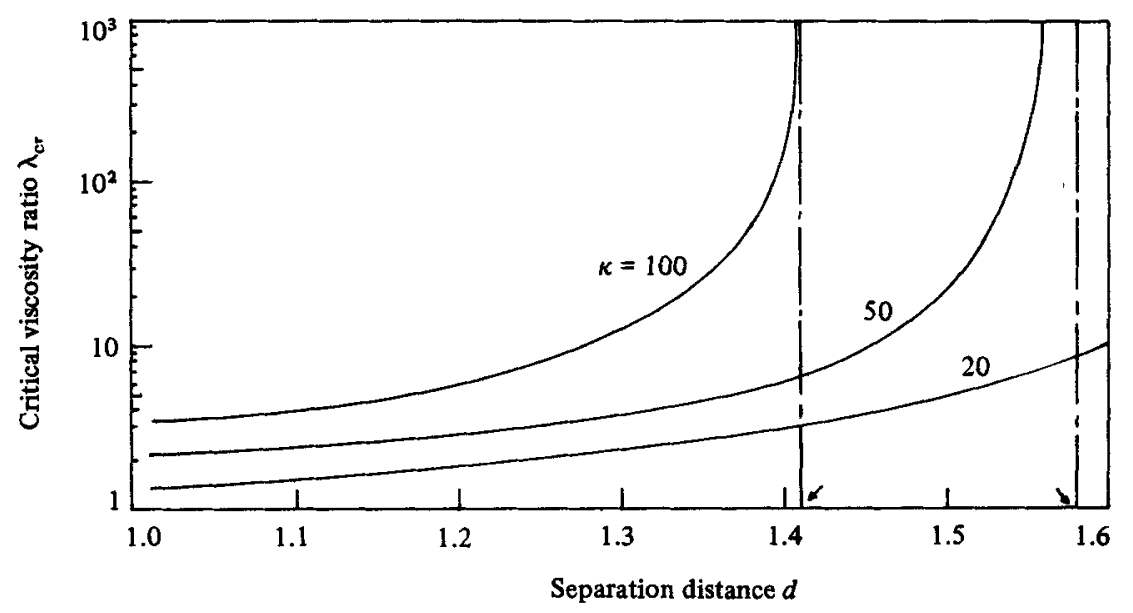

Figure 8. Critical viscosity ratio $\lambda_{\mathrm{er}}$, as a function of the separation distance $d$ for aspect ratios $\kappa=20,50$ and $100 ; \hat{U}_{2}=E \cdot\left(x-x_{p}\right)$ and $S(x)=\frac{1}{2} \ln \left[1-(x / l)^{2}\right]$.

The undisturbed straining flow $\boldsymbol{O}_{i}=\boldsymbol{E} \cdot\left(\boldsymbol{x}-\boldsymbol{x}_{\mathrm{p}}\right)$ is axisymmetric around the $x_{3}$ axis with origin at the body centre $x_{\mathrm{p}}$, and the magnitudes and directions of the total force and torque therefore remain unchanged by rotation of the body around the $x_{3}$ axis (i.e. they are independent of $\phi$-orientation). Indeed, the vector components of the total force and torque for arbitrary $\phi$ can be obtained by simply using $\boldsymbol{Q}^{-1} \cdot \boldsymbol{K}$ for each tensor quantity $K$ in $(24 a, b)$, which is the result for $\phi=0^{\circ}$ (i.e. the $x_{1}$ axis coincides with the projection of the body axis onto the interface).

All of the preceding discussion is concerned with the force and torque on a body in the flow $U_{i}=E \cdot\left(x-x_{p}\right)$ with stagnation point at the body centre. In order to determine the force and torque when the body is located at an arbitrary point $x_{p}$ in the undisturbed flow $\hat{O}_{i}=\boldsymbol{E} \cdot \boldsymbol{x}$, which is zero at the interface, the results of $(24 a, b)$ must be combined with the corresponding results from Part 1 for translation with velocity $-\boldsymbol{E} \cdot \boldsymbol{x}_{\mathrm{p}}$, i.e.

$$
F=-K_{\mathrm{T}} \cdot \boldsymbol{E} \cdot \boldsymbol{x}_{\mathrm{p}}+\boldsymbol{Q}^{-1} \cdot \boldsymbol{K}_{\mathrm{PF}} \cdot \boldsymbol{\xi}_{\mathrm{p}} \quad \text { and } \quad T=-\boldsymbol{K}_{\mathrm{C}} \cdot \boldsymbol{E} \cdot \boldsymbol{x}_{\mathrm{p}}+\boldsymbol{Q}^{-1} \cdot \boldsymbol{K}_{\mathrm{PT}} \cdot \boldsymbol{\xi}_{\mathrm{p}}
$$

The resistance matrices $\boldsymbol{K}_{\mathrm{T}}$ and $\boldsymbol{K}_{\mathrm{C}}$ were determined in Part 1 of this work (Yang \& Leal 1983). The hydrodynamic force and torque $(26 a, b)$ will be used to calculate complete particle trajectories for the general flow $\hat{U}_{2}=E \cdot x$ in $\S 5$.

\subsection{Simple shear flow}

Finally we turn to the case of a slender body in the simple shear flow $\hat{O}_{i}=\left(\mu_{i} / \mu_{2}\right) \boldsymbol{\Gamma} \cdot \boldsymbol{x}$. A general solution for this problem can be obtained by superimposing the results for a uniform translation with velocity $\hat{O}_{i}=\Gamma \cdot x_{\mathrm{p}}$ and a linear shear flow with origin at the body centre, $V_{i}=\boldsymbol{\Gamma} \cdot\left[\left(\mu_{i} / \mu_{2}\right) \boldsymbol{x}-\boldsymbol{x}_{\mathrm{p}}\right]$. Without loss of generality, we assume that the particle is oriented either parallel to the plane of the flow (i.e. $\left.\phi=0^{\circ}, \quad \sigma_{i}=\Gamma_{13}\left[\left(\mu_{i} / \mu_{2}\right) x_{3}+d\right] e_{1}\right)$ or perpendicular to the plane (i.e. $\phi=0^{\circ}$, $\left.O_{i}=\Gamma_{23}\left[\left(\mu_{i} / \mu_{2}\right) x_{3}+d\right] e_{2}\right)$. The solution for an arbitrary $\phi$-orientation can then be determined from the solutions for these two cases using the orthogonal rotation tensor $\boldsymbol{Q}$ which transforms any arbitrary velocity components of $\hat{U}_{i}$ parallel to the interface to components parallel and perpendicular to the plane in which the particle is placed (see figure 4).

First, we consider the case of a slender body with arbitrary $\theta$-orientation, but 
$\phi=0^{\circ}$, in the simple shear flow $G_{i}=\Gamma_{13}\left[\left(\mu_{i} / \mu_{2}\right) x_{3}+d\right] e_{1}$, which vanishes at the body centre. The required Stokeslet and potential dipole distributions along the body centreline to satisfy the boundary condition $(5 b)$ can be determined using the approach outlined in $\$ 4.1$. The result is

$$
\alpha_{1}(x)=-\frac{1}{4}\left(1+\sin ^{2} \theta\right) \sin \theta \Gamma_{13} x\left[\epsilon-\frac{\epsilon^{2}}{2}\left(2 S(x)+\frac{\sin ^{2} \theta-3}{1+\sin ^{2} \theta}+U(x ; \lambda, \theta, d)\right)\right]+O\left(\epsilon^{3}\right)
$$

and

$$
\alpha_{3}(x)=\frac{1}{4} \sin ^{2} \theta \cos \theta \Gamma_{13} x\left[\epsilon-\frac{1}{2} \epsilon^{2}(2 S(x)+1+V(x ; \lambda, \theta, d))\right]+O\left(\epsilon^{3}\right) .
$$

From the Stokeslet distribution we can evaluate the hydrodynamic force and torque on the body (i.e. figure $1 f$ ):

and

$$
\begin{aligned}
& F_{1}=-\epsilon^{2} \pi \sin \theta\left(1+\sin ^{2} \theta\right) \Gamma_{13} \int_{-1}^{1} x U(x ; \lambda, \theta, d) \mathrm{d} x+O\left(\epsilon^{3}\right), \\
& F_{3}=\epsilon^{2} \pi \sin ^{2} \theta \cos \theta \Gamma_{13} \int_{-1}^{1} x V(x ; \lambda, \theta, d) \mathrm{d} x+O\left(\epsilon^{3}\right)
\end{aligned}
$$

$$
\begin{aligned}
T_{2}= & \epsilon \frac{8}{3} \pi \sin ^{2} \theta \Gamma_{13}\left[1-\epsilon\left(\ln 2-\frac{11}{6}\right.\right. \\
& \left.\left.+\frac{3}{8} \int_{-1}^{1}\left[\left(1+\sin ^{2} \theta\right) U(x ; \lambda, \theta, d)+\cos ^{2} \theta V(x ; \lambda, \theta, d)\right] x^{2} \mathrm{~d} x\right)\right]+O\left(\epsilon^{3}\right) .
\end{aligned}
$$

In figures 9 and 10 the force components $F_{1}$ and $F_{3}$ of $(28 a, b)$ are plotted as functions of the orientation angle $\theta$ for $d=1.01$ and 2 . It can be noted from figure 9 that in the flow $O_{2}=\Gamma_{13}\left(x_{3}+d\right) e_{1}$, with origin at the centre of the body axis, the direction of the induced force $F_{1}$, which is obviously zero in an unbounded single fluid, depends on the viscosity ratio $\lambda$ with a degree of sensitivity that is a strong function of the particle position and orientation relative to the interface.

The force component $F_{3}$, which is very small compared with the parallel force $F_{1}$, is a consequence of the asymmetry of particle-interface geometry for $\theta \neq 0,90^{\circ}$ (indeed, the force $F_{3}$ is zero for a sphere). The qualitative features of $F_{3}$ as a function of the orientation angle $\theta$ are, in fact, quite similar for all viscosity ratios $\lambda$ and particle positions relative to the interface. Thus, for $0^{\circ}<\theta<90^{\circ}$, the interface will induce a translation away from the interface in the absence of an applied force $-F_{3}$, while the induced translation would be toward the interface for $90^{\circ}<\theta<180^{\circ}$.

Detailed calculation of the hydrodynamic torque $T_{2}$ given by (28c) shows that the qualitative dependence of $T_{2}$ on the orientation angle $\theta$ is unchanged by the interface. In fact, the effect of the interface becomes very weak when the orientation angle $\theta$ of the body axis is in the range $-30^{\circ} \leqslant \theta \leqslant 30^{\circ}$ (i.e. the effect of the interface on the torque is significant only when one end of the body passes close to the interface).

We have already noted that the existence of the normal force $F_{3},(28 b)$, implies that a freely suspended slender body, in a simple shear flow $\hat{O}_{2}=\Gamma_{13}\left(x_{3}+d\right) e_{1}$ with origin at the body centre, would move in and out relative to the interface as it rotates around the $x_{2}$ axis owing to the hydrodynamic torque $T_{2}$ given by $(28 c)$. However, the trajectory is not periodic, since the torque vanishes in the slender-body approximation at $\theta=n \pi$, and the body is predicted to experience a net outward displacement relative to the interface from its initial position. Comparison with existing theoretical results for a slender body in simple shear flow of a single unbounded fluid suggests strongly that this non-periodicity in the particle motion is a consequence of the slender-body approximation. In particular, Cox (1971) showed that the force and 


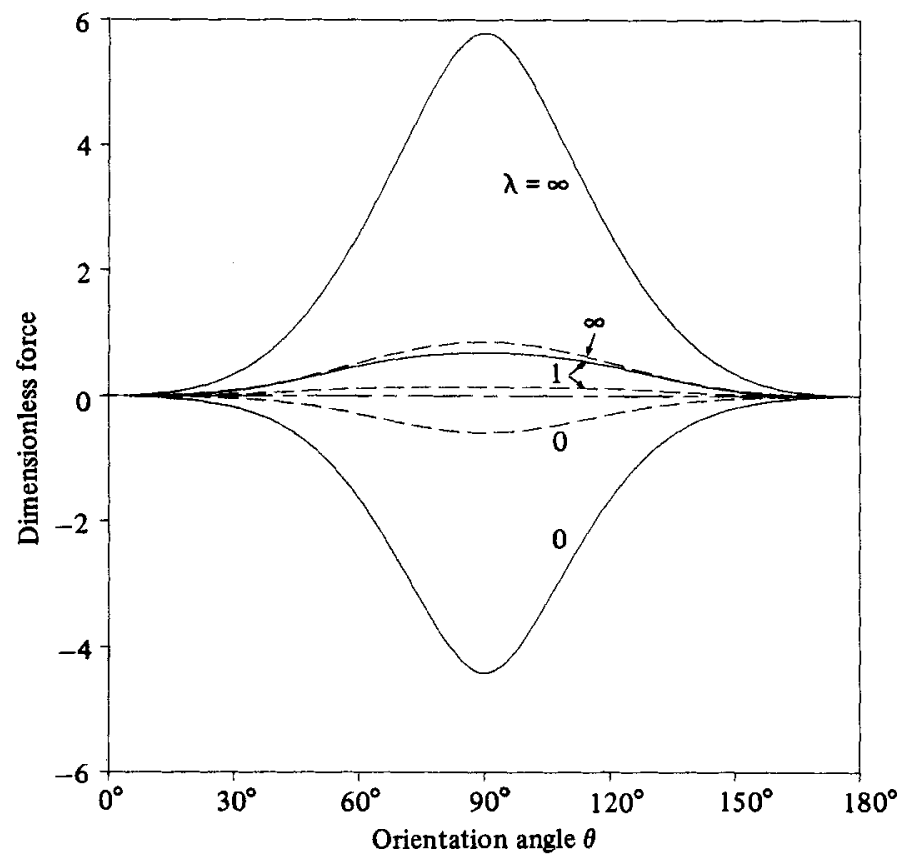

Figure 9. Dimensionless force $F_{1} / \epsilon^{2} \mu_{2} \Gamma_{13} l^{2}$ of $(28 a)$ as a function of the orientation angle $\theta ; \hat{U}_{2}=\Gamma_{13}\left(x_{3}+d\right) e_{1}, \epsilon=0.1887, S(x)=\frac{1}{2} \ln \left[1-(x / l)^{2}\right]:-, d=1.01 ;-\longrightarrow-, 2.0$; unbounded-fluid case.

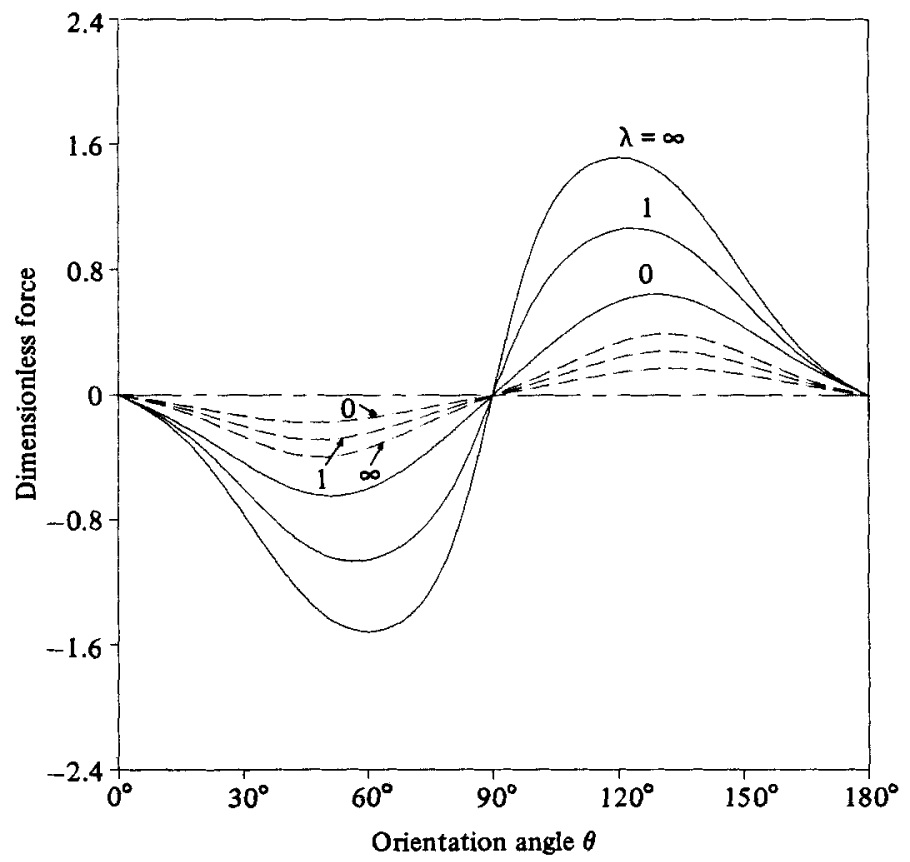

Figure 10. Dimensionless force $F_{3} / \epsilon^{2} \mu_{2} \Gamma_{13} l^{2}$ of $(28 b)$ as a function of the orientation angle $\theta ; O_{2}=\Gamma_{13}\left(x_{3}+d\right) e_{1}, \epsilon=0.1887, S(x)=\frac{1}{2} \ln \left[1-(x / l)^{2}\right]:-, d=1.01 ;-\ldots, 2.0 ;-\square$, unbounded-fluid case. 
torque on an axisymmetric slender body that is at rest and oriented parallel to a simple shear flow $(\theta=n \pi)$ is $O\left((1 / \kappa)^{2} \epsilon\right)$, which is very small compared with the $O\left(\epsilon^{2}\right)$ terms retained in $(28 a-c)$, but is definitely non-zero. According to Cox's analysis, a slender body will rotate very slowly through the aligned, or nearly aligned, state, but will experience a periodic rotation for any large (but finite) $\kappa$. Similar behaviour in the present problem of particle motion near an interface would imply that any real particle (with finite $\kappa$ ) would both rotate and move in and out continuously. We shall return shortly to the details of this motion, which is a generalization of the famous Jeffery (1922) orbit for rotation in simple shear flow of a single unbounded fluid.

Now, let us turn to the hydrodynamic interface effects on a slender body in the simple shear flow $\hat{O}_{i}=\Gamma_{23}\left[\left(\mu_{i} / \mu_{2}\right) x_{3}+d\right] e_{2}$, which is perpendicular to the plane defined by the body axis and normal vector $e_{3}$ to the interface. In this case, the boundary condition at the body surface $(5 b)$ is

$$
u_{2}\left(x_{\mathrm{B}}\right)=-\Gamma_{23} x \sin \theta e_{2}+O\left(\frac{1}{\kappa}\right) \quad\left(x_{\mathrm{B}} \in S_{\mathrm{p}}\right)
$$

It may be noted, however, that this boundary condition is exactly the same as for particle rotation near a flat interface with angular velocity $\boldsymbol{\Omega}=\Omega_{1} \boldsymbol{e}_{1}$ through a fluid at rest at infinity, with $\Omega_{1}=\Gamma_{23}$. Equations for the hydrodynamic force and torque in this latter case have already been derived by Yang \& Leal (1983).

We now have a complete solution for a slender body in a simple shcar flow $\hat{U}_{i}=\Gamma \cdot\left[\left(\mu_{i} / \mu_{2}\right) x-x_{\mathrm{p}}\right]$ with origin at the body centre and the undisturbed velocity either parallel or perpendicular to the plane defined by the body centreline and normal vector $e_{3}$ to the interface. From these results we can also evaluate the force and torque on a slender body with an arbitrary orientation $(\theta, \phi)$ located at an arbitrary position $\boldsymbol{x}_{\mathrm{p}}$ in a simple shear flow $\hat{U}_{i}=\left(\mu_{i} / \mu_{2}\right) \boldsymbol{\Gamma} \cdot \boldsymbol{x}$ with origin at the interface. Combining the results of the present section with those for uniform streaming flow, we obtain

and

$$
F=-K_{\mathrm{T}} \cdot \Gamma \cdot x_{\mathrm{p}}+Q^{-1} \cdot K_{\mathrm{SF}} \cdot Q \cdot \xi_{\mathrm{s}}
$$

$$
\boldsymbol{T}=-\boldsymbol{K}_{\mathrm{C}} \cdot \boldsymbol{\Gamma} \cdot \boldsymbol{x}_{\mathrm{p}}+\boldsymbol{Q}^{-1} \cdot \boldsymbol{K}_{\mathrm{ST}} \cdot \boldsymbol{Q} \cdot \boldsymbol{\xi}_{\mathrm{s}}
$$

Here the non-zero components of hydrodynamic tensors $\boldsymbol{\kappa}_{\mathrm{SF}}$ and $\boldsymbol{K}_{\mathrm{ST}}$ are given by

$$
\begin{aligned}
& K_{\mathrm{SF}}^{11}=-\epsilon^{2} \pi\left(1+\sin ^{2} \theta\right) \sin \theta \int_{-1}^{1} x U(x ; \lambda, \theta, d) \mathrm{d} x+O\left(\epsilon^{3}\right) \\
& K_{\mathrm{SF}}^{22}=-\epsilon^{2} 2 \pi \sin \theta \int_{-1}^{1} x B(x ; \lambda, \theta, d) \mathrm{d} x+O\left(\epsilon^{3}\right) \\
& K_{\mathrm{SF}}^{31}=\epsilon^{2} \pi \sin ^{2} \theta \cos \theta \int_{-1}^{1} x V(x ; \lambda, \theta, d) \mathrm{d} x+O\left(\epsilon^{3}\right) \\
& K_{\mathrm{ST}}^{12}=-\epsilon \frac{8}{3} \pi \sin ^{2} \theta\left[1-\epsilon\left(\ln 2-\frac{11}{6}+\frac{3}{4} \int_{-1}^{1} x^{2} K(x ; \lambda, \theta, d) \mathrm{d} x\right)\right]+O\left(\epsilon^{3}\right) \\
& K_{\mathrm{ST}}^{21}=\epsilon \frac{8}{3} \pi \sin ^{2} \theta\left[1-\epsilon\left(\ln 2-\frac{11}{6}+\frac{3}{8} \int_{-1}^{1}\left(\left(1+\sin ^{2} \theta\right) U(x ; \lambda, \theta, d)\right.\right.\right. \\
&\left.\left.\left.\quad+\cos ^{2} \theta V(x ; \lambda, \theta, d)\right) x^{2} \mathrm{~d} x\right)\right]+O\left(\epsilon^{3}\right)
\end{aligned}
$$

and

$$
K_{\mathrm{ST}}^{32}=-K_{\mathrm{ST}}^{12} \cot \theta .
$$

Specific formulae for $U(x ; \lambda, \theta, d), V(x ; \lambda, \theta, d), K(x ; \lambda, \theta, d)$ and $B(x ; \lambda, \theta, d)$ are given in the Appendix.

We now have a complete set of solutions either for a stationary sphere or slender 
body located at an arbitrary point $x_{p}$ with an arbitrary orientation relative to the interface in either an axisymmetric pure extensional flow, or in a simple shearing flow field. These solutions provide the necessary relationships between the flow parameters (e.g. strain rate or shear rate) and the hydrodynamic force and torque for calculation of particle trajectories, which we shall consider in $\$ 5$.

\section{Trajectories near a flat interface}

Whenever the creeping-motion approximation is applicable, general relationships can be written between the force and torque acting on a particle in a quiescent fluid near a flat interface, and its translational and angular velocities in terms of

$$
\boldsymbol{F}_{\mathrm{ex}}=\boldsymbol{K}_{\mathrm{T}} \cdot \boldsymbol{U}+\boldsymbol{K}_{\mathrm{C}}^{\mathrm{t}} \cdot \boldsymbol{\Omega}, \quad \boldsymbol{T}_{\mathrm{ex}}=\boldsymbol{K}_{\mathrm{C}} \cdot \boldsymbol{U}+\boldsymbol{K}_{\mathrm{R}} \cdot \boldsymbol{\Omega},
$$

the so-called hydrodynamic resistance tensors $\boldsymbol{K}_{\mathrm{T}}, \boldsymbol{K}_{\mathrm{R}}$ and $\boldsymbol{K}_{\mathrm{C}}$. The components of these tensors for a spherical particle were evaluated through terms $O\left(\delta^{2}\right)$ by Lee et al. (1979), and through terms $O\left(\delta^{3}\right)$ in the present study $(11 a-e)$. For a slender body, Yang \& Leal (1983) obtained the various components of these tensors up to $O\left(\epsilon^{2}\right)$.

In the present paper we consider only the simplest case of a neutrally buoyant freely suspended body. In this case, the translational and angular velocities of the particle are given by

$$
\begin{aligned}
& \boldsymbol{U}=\frac{\mathrm{d} \boldsymbol{x}_{\mathrm{p}}}{\mathrm{d} t}=\left(\boldsymbol{K}_{\mathrm{T}}-\boldsymbol{K}_{\mathrm{C}}^{\mathrm{t}} \cdot \boldsymbol{K}_{\mathrm{R}}^{-1} \cdot \boldsymbol{K}_{\mathrm{C}}\right)^{-1} \cdot\left(\boldsymbol{F}-\boldsymbol{K}_{\mathrm{C}}^{\mathrm{t}} \cdot \boldsymbol{K}_{\mathbf{R}}^{-1} \cdot \boldsymbol{T}\right), \\
& \boldsymbol{\Omega}=\left(\boldsymbol{K}_{\mathrm{R}}-\boldsymbol{K}_{\mathrm{C}} \cdot \boldsymbol{K}_{\mathrm{T}}^{-1} \cdot \boldsymbol{K}_{\mathrm{C}}^{\mathrm{t}}\right)^{-1} \cdot\left(\boldsymbol{T}-\boldsymbol{K}_{\mathrm{C}} \cdot \boldsymbol{K}_{\mathrm{T}}^{-1} \cdot \boldsymbol{F}\right) .
\end{aligned}
$$

Here $\boldsymbol{F}$ and $\boldsymbol{T}$ are the hydrodynamic force and torque acting on a stationary particle due to the existence of a pure straining or simple shearing flow at large distance from the particle. Thus, given the initial position and orientation of the particle, these equations provide its complete trajectory (i.e. its position and orientation as a function of time). In the present work, we use a simple Runge-Kutta scheme described by Yang \& Leal (1983) to integrate $(33 a, b)$.

\subsection{Trajectories of a sphere}

First, we begin with the case of a neutrally buoyant sphere freely suspended in the pure straining flow $\hat{O}_{i}=\boldsymbol{E} \cdot \boldsymbol{x}$ with stagnation point at the interface. The results for the torque and force $F$ and $T$ in this case are given in $(10 a, b)$. Substituting for $F$ and $T$ in (33), it is a simple matter to show that the translational and angular velocities of the particle are

$$
\boldsymbol{U}=\boldsymbol{E} \cdot \boldsymbol{x}_{\mathrm{p}}-\frac{\frac{5}{16} \delta^{2} \frac{2+3 \lambda}{1+\lambda}\left[1+\frac{3}{8} \delta \frac{2+3 \lambda}{1+\lambda}\right]}{\left[1+\sum_{n=1}^{3}\left(\frac{3}{8} \delta \frac{2+3 \lambda}{1+\lambda}\right)^{n}-\frac{1+4 \lambda}{8(1+\lambda)} \delta^{3}\right]} e_{3} \text { and } \Omega=0 . \quad(34 a, b)
$$

Thus the particle does not rotate at all, at the level of approximation represented by $(34 a, b)$, and it is only the $U_{3}$ component of the translational velocity that is altered from the undisturbed velocity of the fluid by the presence of an interface.

It can be noted from $(34 a)$ that the particle velocity $U_{3}$ is always decreased in magnitude by the presence of an interface, independently of the viscosity ratio $\lambda$. Further, the difference between $U_{3}$ and the undisturbed velocity of the fluid $\boldsymbol{E} \cdot \boldsymbol{x}_{\mathrm{p}} \cdot \boldsymbol{e}_{3}$ is monotonically increased as the separation between the interface and sphere is decreased, but is independent of the distance from the axis of symmetry of the undisturbed flow. 


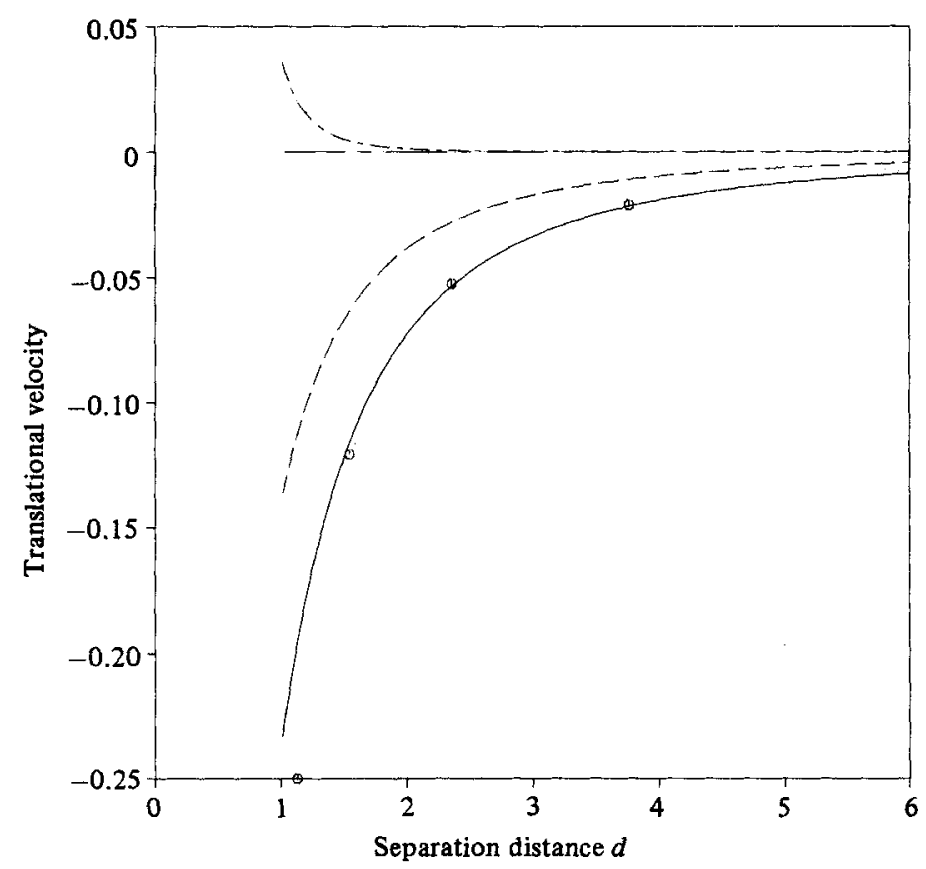

Figure 11. Dimensionless disturbed translational velocity $\left(U_{1}-\Gamma_{13} d\right) / \Gamma_{13} a$ as a function of the dimensionless distance $d$ between the sphere centre and the interface; $\sigma_{2}=\Gamma_{13} x_{3} e_{1}$ : $\lambda=\infty ; \ldots \ldots, 1, \ldots \ldots, 0, \ldots \ldots, \ldots, \ldots$, unbounded-fluid case; 0 , corresponding exactsolution results of Goldman et al. (1967b).

The motion of a sphere in a linear shear flow $V_{i}=-\left(\mu_{i} / \mu_{2}\right) \Gamma_{13} x_{3} e_{1}$, parallel to the interface can be resolved in a similar manner. Since the hydrodynamic force on the sphere is oriented parallel to the undisturbed flow, i.e. $F=F_{1} e_{1}$ (cf. $(17 a)$ ), the path followed by the sphere in the $\left(x_{1}, x_{3}\right)$-plane is exactly coincident with a streamline of the undisturbed flow. However, the translational velocity of the sphere is altered considerably from the undisturbed velocity of the fluid by interaction with the interface. This is illustrated in figure 11, where the difference between the velocity of the sphere and the undisturbed velocity of the fluid $\left(U_{1}-\Gamma_{13} d\right) / \Gamma_{13} a$ is given as a function of the separation distance $d$ between the sphere and the interface for three values of $\lambda=0,1$ and $\infty$. Also included for comparison are the corresponding results of Goldman et al. $(1967 b)$, who obtained an exact solution of the Stokes equations, using bipolar coordinates, for the translational and angular velocities of a neutrally buoyant sphere moving in a linear shear flow in proximity to a single plane wall (i.e. $\lambda \rightarrow \infty$ ). It can be seen from figure 11 that the present asymptotic result for the translational velocity is in reasonable agreement with the exact solution in the entire region of $d>1$. Indeed, the relative error associated with the asymptotic solution is less than $2.0 \%$ for $d>1.54$.

The angular velocity $-\Omega_{2},(33 b)$, for motion of a freely suspended sphere in the simple shearing flow is plotted in figure 12 as a function of $d$ for three values of $\lambda=0$, 1 and $\infty$. Darabaner \& Mason (1967) experimentally measured the angular velocity of a neutrally buoyant sphere in a Couette viscometer as a function of the separation distance between the sphere and the wall of the viscometer. Their results are included in the figure. In addition, the exact solution of Goldman et al. $(1967 b)$ for $\lambda=\infty$ is also compared with our approximate solution in this figure. The present asymptotic 


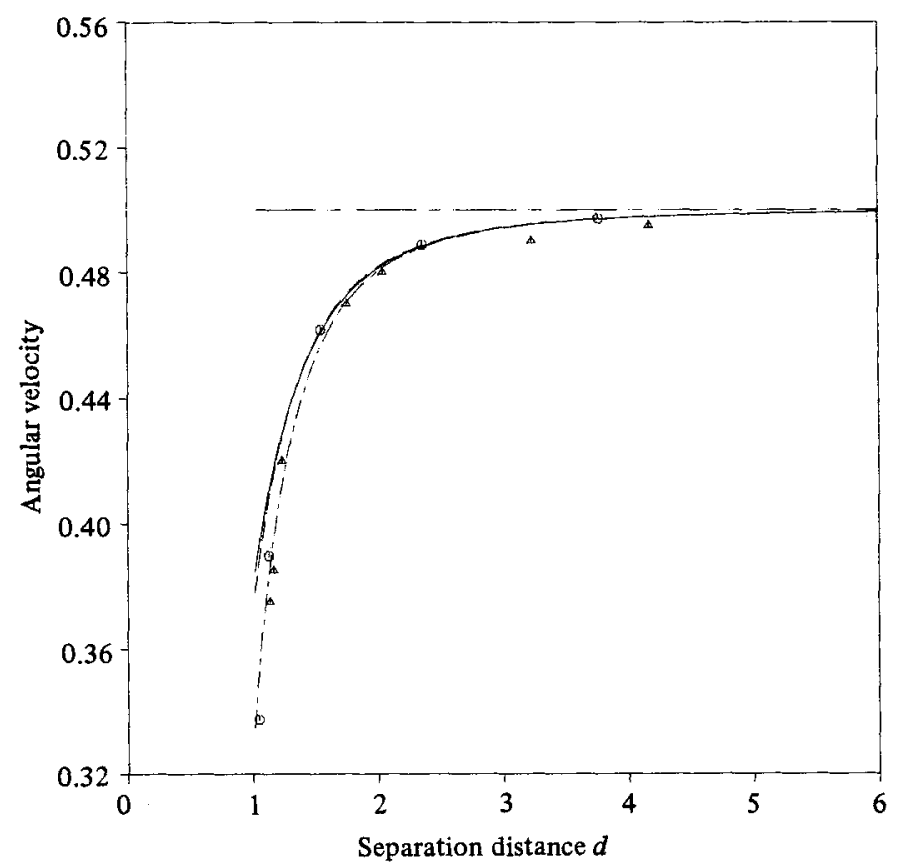

FIgURe 12. Dimensionless angular velocity $-\Omega_{2} / \Gamma_{13}$ as a function of the dimensionless distance $d$ between the sphere centre and the interface; $O_{2}=\Gamma_{13} x_{3} e_{1}:-, \lambda=\infty ;---, 1 ;--\cdots$, $\lambda=0$; — unbounded-fluid case; $O$, corresponding exact-solution results of Goldman et al. $(1967 b) ; \triangle$, experimental data of Darabaner \& Mason (1967).

solution is qualitatively consistent both with the experimental data and the exact solution over the whole range of $d$, and is quantitatively accurate except in the region $d \sim 1$. Considering that the experimental data have neither been corrected for wall curvature nor for the presence of a second wall at a larger distance, and in view of the difficulties of maintaining and measuring the separation distance from the wall, the agreement is quite good.

\subsection{Trajectories of a slender body}

Let us turn now to the case of a slender body suspended freely in a linear flow field. Since each hydrodynamic resistance tensor in $(33 a, b)$ is a function of the orientation of the body axis $(\theta, \phi)$, in addition to the position of the body relative to the interface (i.e. $d$ ), it is convenient to relate the angular velocity $\Omega$ in $(33 b)$ to $\dot{\theta}$ and $\dot{\phi}$, the time rate of changes in $\theta$ and $\phi$ (cf. Yang \& Leal 1983).

We begin with the trajectory of a slender body in the pure straining flow $\hat{O}_{i}=\boldsymbol{E} \cdot \boldsymbol{x}$. As we noted in $\$ 4.1$, the hydrodynamic torque on the body in this flow is due primarily to the basic flow rather than the interaction between the particle and the interface. Only for $\lambda \geqslant O(1)$ with $d-l \ll O(1)$ and $\theta$ in the range $45^{\circ}-135^{\circ}$, so that one end of the particle is relatively close to the interface, is there a significant contribution to the torque from the particle-interface interaction (cf. figures 7 and 8). We now thus consider a slender body initially located at $x_{\mathrm{p}}^{0}=(0,0,-2)$, which is relatively close to the interface, with initial oblique angles $\theta_{0}=0^{\circ}, 30^{\circ}, 60^{\circ}, 70^{\circ}, 75^{\circ}, 85^{\circ}$ and $90^{\circ}$ relative to the interface and $\phi_{0}=0^{\circ}$. In this case $\left(\phi_{0}=0^{\circ}\right)$, the axis of the particle is initially in the $\left(x_{1}, x_{3}\right)$-plane, and remains so as it travels along the flow field. In figure 13 the trajectories for a slender body with preseribed initial position and 


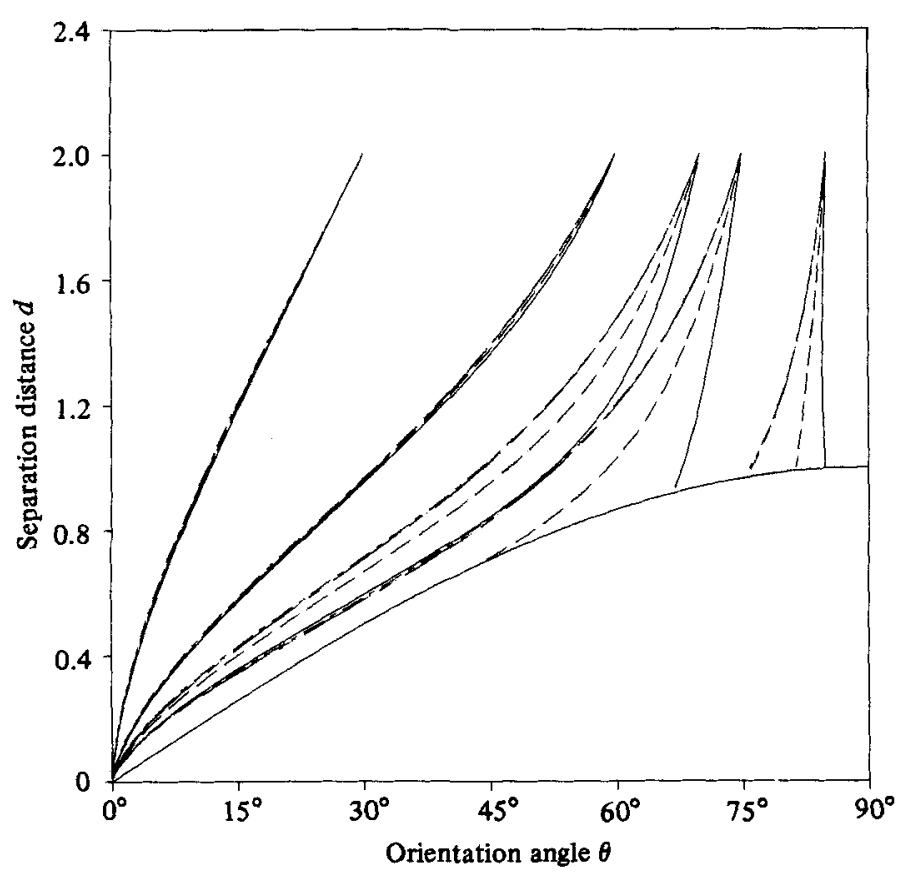

Figure 13. Trajectories for a neutrally buoyant slender body in a pure straining flow $\boldsymbol{U}_{2}=E \cdot x$, in terms of the $\theta$ and $d ; \phi_{0}=0^{\circ}, \theta_{0}=0^{\circ}, 30^{\circ}, 60^{\circ}, 70^{\circ}, 75^{\circ}, 85^{\circ}$ and $90^{\circ}, \epsilon=0.1883, S(x)=\frac{1}{2} \ln \left[1-(x / l)^{2}\right]$; $\longrightarrow, \lambda=\infty ;-\ldots, \lambda=1 ; \ldots \ldots, 0, \ldots, \ldots, \ldots$

orientations are represented in terms of the orientation angle $\theta$ and the separation distance $d$ for three values of $\lambda=0,1$ and $\infty$. We also include the corresponding results for trajectories in an unbounded fluid, which nearly coincide with those for the $\lambda=0$ case. A slender body initially oriented parallel or perpendicular to the interface will travel along the flow without rotation, and thus the trajectory ( $d$ versus $\theta$ ) in each case is a vertical straight line. Furthermore, for any initial orientation $\theta_{0}$, except the case of $\lambda=\infty$ and $\theta_{0}=85^{\circ}$, the particle always rotates towards an orientation parallel to the interface independently of $\lambda$. For the case of $\lambda=\infty$ and $\theta_{0}=85^{\circ}$, on the other hand, the particle rotates towards the perpendicular orientation, which, as we have noted earlier, is a second stable equilibrium orientation for this case. The final orientation for $\lambda=\infty$ is determined by the initial position and orientation of the particle. This rather curious result for $\lambda=\infty$ will actually occur for any value of $\lambda>\lambda_{\mathrm{cr}}$, which is determined from figure 8. It may be noted that a slender body with initial orientation $\theta_{0} \leqslant 70^{\circ}$ achieves an orientation parallel to the interface before the particle reaches the interface (actually up to $d / l-|\sin \theta|=0.01$, which is the separation distance between the tip of the body and the interface). On the other hand, a particle with $\theta_{0} \leqslant 75^{\circ}$, except the case of $\lambda=0$ and $\theta_{0}=75^{\circ}$, touches the interface before it arrives at the equilibrium orientation either parallel or perpendicular to the interface, depending on the viscosity ratio. The critical value of the initial orientation $\theta_{0}$, determining the final orientation, depends on the viscosity ratio $\lambda$ and the initial separation from the interface. However, a particle initially located at sufficiently large distance (i.e. $d \rightarrow \infty)$ with arbitrary orientation $\left(\theta \neq 90^{\circ}\right)$ will always rotate parallel to the interface before it reaches the interface.

The trajectories for other initial positions $x_{p}^{0}=(0.5,0,-2),(1,0,-2),(3,0,-2)$ and $(5,0,-2)$, which are displaced from the axis of symmetry, were also examined. 
The trajectories in the $\left(x_{1}, x_{3}\right)$-plane (i.e. $d$ versus $x$ ) do not deviate significantly from the corresponding streamlines of the undisturbed flow field. Furthermore, the hydrodynamic torque on the particle in the flow, i.e. $T-K_{\mathrm{C}} \cdot K_{\mathrm{T}}^{-1} \cdot \boldsymbol{F}$ from $(33 b)$ in combination with $(26 a, b)$, equals $\boldsymbol{Q}^{-1} \cdot \boldsymbol{K}_{\mathrm{PT}} \cdot \boldsymbol{\xi}_{\mathrm{p}}-\boldsymbol{K}_{\mathrm{C}} \cdot \boldsymbol{K}_{\mathrm{T}}^{-1} \cdot \boldsymbol{Q}^{-1} \cdot \boldsymbol{K}_{\mathrm{PF}} \cdot \boldsymbol{\xi}_{\mathrm{p}}$, which depends on the separation distance $d$ from the interface and the particle orientation $(\theta, \phi)$, but is independent of the particle position relative to the axis of symmetry. The terms $\boldsymbol{Q}^{-1} \cdot \boldsymbol{K}_{\mathrm{PT}} \cdot \boldsymbol{\xi}_{\mathrm{p}}$ and $\boldsymbol{Q}^{-1} \cdot \boldsymbol{K}_{\mathrm{PF}} \cdot \boldsymbol{\xi}_{\mathrm{p}}$ are the hydrodynamic torque and force on a particle in the pure straining flow that has its origin coincident with position of the particle centre, while $\boldsymbol{K}_{\mathrm{C}} \cdot \boldsymbol{K}_{\mathrm{T}}^{-1} \cdot \boldsymbol{Q}^{-1} \cdot \boldsymbol{K}_{\mathrm{PF}} \cdot \xi_{\mathrm{p}}$ is the torque acting on the same particle as a consequence of force $\boldsymbol{Q}^{-1} \cdot \boldsymbol{K}_{\mathrm{PF}} \cdot \boldsymbol{\xi}_{\mathrm{p}}$ and the reciprocity of Stokes flow with linear boundary conditions. Thus the angular velocity, $(33 b)$, of a slender body located at arbitrary point $\boldsymbol{x}_{\mathrm{p}}^{\mathbf{0}}$ is determined by the separation $d$ from the interface, for a given orientation $(\theta, \phi)$, and is independent of the particle position relative to the axis of symmetry. The general features of the particle trajectories in terms of the orientation $\theta$ versus the separation distance $d$, which were described for $x_{p}^{0}=(0,0,-2)$, are therefore preserved whether or not the initial location is on the axis of symmetry, at least for the special initial separation distance, i.e. $d=2$, considered here.

The other problem that we examine in this section is an undisturbed simple shearing flow, $\hat{O}_{2}=-x_{3} e_{1}$, parallel to the interface into which a slender ellipsoid of revolution (i.e. $S(x)=0$ ) is placed with an arbitrary orientation determined by spherical polar angles $\theta$ and $\phi$ based upon the plane of the interface (ef. figure 4). If the axis ratio for the ellipsoid is arbitrarily small, but non-zero, and the ellipsoid is suspended freely in simple shear flow of an unbounded single fluid, Jeffery (1922) showed that the motion of the axis of revolution of the particle is described, apart from a simple translation parallel to the flow, by periodic (Jeffery) orbit equations, relating $\dot{\theta}$ and $\dot{\phi}$ to $\kappa, \theta$ and $\phi$. The corresponding equations for slender-body rotation in an unbounded single fluid can be calculated readily for the present slender-body solution of $O\left(\epsilon^{2}\right)$ by using (30) and (33):

$$
\dot{\theta}=\cos \phi \sin ^{2} \theta(1-0.5 \epsilon)+O\left(\epsilon^{2}\right), \quad \dot{\phi}=\sin \phi \tan \theta(1-0.5 \epsilon)+O\left(\epsilon^{2}\right)
$$

In the limit $\kappa \rightarrow \infty$ (or $\epsilon \rightarrow 0$ ), the exact and slender-body results are identical except for $\theta \approx n \pi$ (where $n$ is any non-negative integer), when the exact equations yield

$$
\dot{\theta} \approx \cos \phi \kappa^{-2}
$$

while the slender-body approximation reduces to $\dot{\theta}=\dot{\phi}=0$.

In an unbounded fluid, particles with an arbitrarily large but finite aspect ratio $\kappa$ thus rotate periodically through the aligned (or nearly aligned) orientation, $\theta=n \pi$, owing to the small $O\left(\kappa^{-2}\right)$ term of (36), while the slender-body theory predicts that the particles asymptotically approach the aligned position, but do not continue to rotate. Thus, although successful in giving the hydrodynamic resistance for non-aligned orientations (i.e. $\theta \neq n \pi$ ), the classical slender-body theory fails to give any results for the fully aligned state, and this is a critical failure for adequate description of the periodic orbital motion in simple shear flow. This problem was considered in detail for a slender body in a single unbounded fluid by Cox (1971). Cox determined the hydrodynamic torque acting on the slender body with aligned orientation, $\theta=n \pi$, in a linear shearing flow of a single, unbounded fluid as an asymptotic expansion in terms of $1 / \kappa$, i.e.

$$
T=\frac{8 \pi}{3} \frac{\cos \phi \kappa^{-2} \epsilon}{1-0.5 \epsilon}+O\left(\kappa^{-3}\right)
$$

which is responsible for the slow rotation of a real particle through the aligned 
orientations $\theta \sim n \pi$. From (37) and the hydrodynamic relationship between the torque and the angular velocity, we can readily evaluate the angular velocity $\dot{\theta}$ through the aligned orientation $(\theta=n \pi)$.

$$
\dot{\theta}=\cos \phi \kappa^{-2}\left(1+0.25 \epsilon^{2}\right)+O\left(\kappa^{-3} \epsilon^{3}\right),
$$

which is consistent with the exact Jeffery-orbit equation with $\theta=n \pi$, i.e. (36). Leal (1975) has shown that a useful and uniformly valid first approximation to the orbit equation in an unbounded single-fluid case can be obtained simply by combining the first-order slender-body solutions $O(\epsilon)$ with the expression (36) in the form

$$
\dot{\theta} \sim \cos \phi\left(\sin ^{2} \theta+\kappa^{-2}\right) \text { and } \dot{\phi} \sim \sin \phi \tan \theta .
$$

and that the detailed orbit shapes corresponding to $(39 a, b)$ are nearly identical with the famous (and exact) Jeffery orbits. We now examine the trajectories of a slender body in simple shearing flow near a plane interface, using the same approximation $(38)$ to describe rotation $\dot{\theta}$ of the body axis through the aligned orientations near $\theta \sim n \pi$.

First, we begin with the motion of a particle from an initial $\phi$-orientation, $\phi_{0}=0^{\circ}$, in which the axis of the particle is in the $\left(x_{1}, x_{3}\right)$-plane defined by the flow direction and the normal to the interface. Thus the slender body remains always in the plane $\phi=0^{\circ}$ and it is only $\theta$ and the position of the particle centre that change with time. In figure 14 the trajectories for a slender body located initially at $x_{p}^{0}=(0,0,-1.2)$ with initial $\theta$-orientations $\theta_{0}=-30^{\circ}, 0^{\circ}, 10^{\circ}$ and $50^{\circ}$ are represented in terms of the increment $\theta-\theta_{0}$ of the orientation angle and the separation distance $d$ between the body centre and the interface for three values of $\lambda=0,1$ and $\infty$. For an unbounded fluid, the $e_{3}$ component of the hydrodynamic force $F-K_{\mathrm{C}}^{\mathrm{t}} \cdot K_{\mathbf{R}}^{-1} \cdot T$ in $(33 a)$ is obviously zero (cf. figure 10), and thus the trajectory, $d$ versus $\theta$, in that case is a horizontal line regardless of the initial $\theta$-orientation. Here $\boldsymbol{F}$ denotes the hydrodynamic force acting on the slender body in the simple shearing flow without rotation, $(30 a)$, and $T$ is the hydrodynamic torque on the same particle without translation, $(30 \mathrm{~b})$. The trajectories ( $d$ versus $\theta$ ) represented in figure 14 show, however, somewhat-complicated features in the presence of an interface. The present theoretical results show that the hydrodynamic force, $\boldsymbol{F}-\boldsymbol{K}_{\mathrm{C}}^{\mathrm{t}} \cdot \boldsymbol{K}_{\mathrm{R}}^{-1} \cdot \boldsymbol{T}$ in $(33 a)$, induced by the flow field yields not only translation of the body parallel to the interface but also translation towards or away from the interface with a simultaneous rotation in the direction of increasing $\theta$, so that the leading edge turns towards the interface. Although the hydrodynamic force is at equilibrium in the $x_{3}$-direction at each extremum point in figure 14 , the particle orientation changes (i.e. $\theta$ is increased) owing to the non-zero torque $\boldsymbol{T}-\boldsymbol{K}_{\mathrm{C}} \cdot \boldsymbol{K}_{\mathrm{T}}^{-1} \cdot \boldsymbol{F}$ in (33b). Thus the equilibrium cannot be maintained, and the body continues to move in and out relative to the interface as it translates continuously parallel to the interface with a simultaneous rotation. When the particle becomes parallel $\left(\theta=0^{\circ}\right.$ or $180^{\circ}$ ) to the interface (which corresponds to the steepest peak point in the trajectory for each initial $\theta_{0}$ orientation considered here), it begins to travel along the symmetrical trajectory with respect to $\theta=0^{\circ}$ (or $180^{\circ}$ ), as it rotates very slowly through alignment. It is worth pointing out that, owing to the symmetry of the system, the trajectories are exactly symmetrical with respect to $\theta=0^{\circ}$ (or $180^{\circ}$ ), as the particle rotates very slowly through the aligned state. The trajectories are also exactly symmetrical with respect to $\theta=\frac{1}{2} n \pi$ ( $n$; an integer), and thus the particle eventually reaches the initial separation distance from the interface (i.e. in this case $d=1.2$ ) at the orientation angle $\theta=\pi+\theta_{0}$, beyond which the body passes along the same periodic trajectories as those shown in figure 14 . 


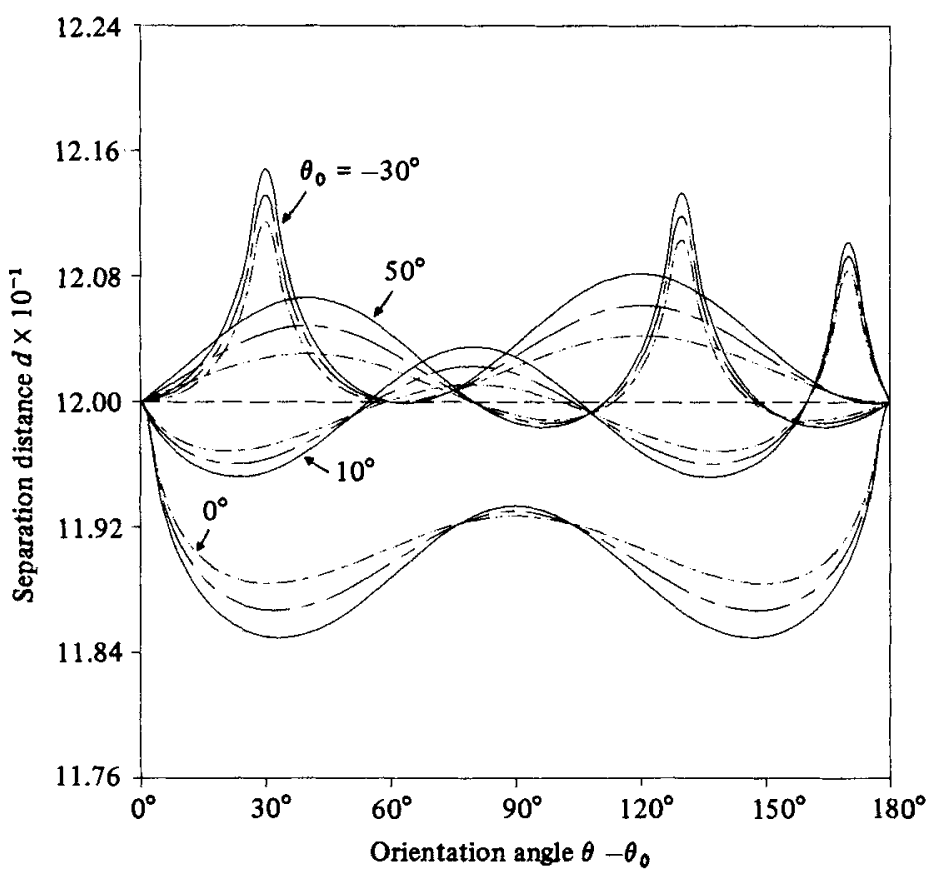

Figure 14. 'Trajectories for a neutrally buoyant slender body in a simple shear flow $\boldsymbol{O}_{2}=\Gamma_{13} x_{3} e_{1}$ in terms of the separation distance $d$ and the orientation angle $\theta ; \phi_{0}=0^{\circ}, \theta_{0}=-30^{\circ}, 0^{\circ}, 10^{\circ}, 50^{\circ}$,

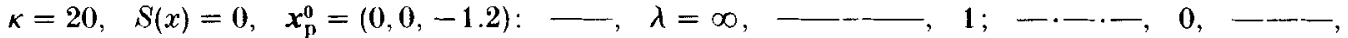
unbounded-fluid case.

Finally, we consider the case of a slender body initially oriented with $\phi_{0} \neq 0^{\circ}$ and the same $\theta_{0}$ considered in the foregoing case. In the case $\phi_{0} \neq 0^{\circ}$ the body ax is is no longer in the plane of the flow defined by the flow direction $e_{1}$ and the normal to the interface $e_{3}$, and the trajectories are different from those in figure 14, in which $\phi_{0}=0^{\circ}$. In figure 15 we compare the detailed particle rotation for $\phi_{0}=0^{\circ}, \frac{1}{48} \pi, \frac{1}{36} \pi, \frac{1}{24} \pi$ and $\frac{1}{12} \pi$, as indicated by the projection of the end of the particle onto the plane of the shear flow in a frame of reference fixed to the body centre. The various orbital trajectories for different values of $\phi_{0}$ indicate that the precise projection is quite sensitive to the initial orientation $\left(\theta_{0}, \phi_{0}\right)$. Most clearly evident, on comparing the calculated orbits, is the fact that the general features of trajectories in figure 15 are preserved whether or not the interface is introduced, and the orbital motion is periodic independently of the viscosity ratio $\lambda$ and the initial orientation of the body axis. Indeed, the orbital trajectories for $\lambda=1$ in figure 15 are almost identical with those in an unbounded single fluid. However, the origin of figure 15 (i.e. the body centre) in the presence of an interface periodically oscillates relative to the interface. Thus the trajectories in terms of the separation distance $d$ from the interface and the orientation angle $\theta$ are significantly different from the case of $\phi_{0}=0^{\circ}$, in which the angle $\theta$ is continuously increased as the body rotates. In figure 16, the orbital trajectories for one period in the plane of $d$ versus $\theta$ are plotted for $\phi_{0}=30^{\circ}$ and $\theta_{0}=-30^{\circ}, 0^{\circ}, 10^{\circ}, 50^{\circ}$ and for three values of $\lambda=0,1$ and $\infty$ to illustrate the effect of the initial $\phi$-orientation on the particle motion. Also shown for comparison are the results for an unbounded infinite fluid, in which the trajectory ( $d$ versus $\theta$ ) is the horizontal line. In this case of $\phi_{0} \neq 0^{\circ}$ the body not only tumbles end-to-end but also twists relative to the plane of the flow (i.e. the $\left(x_{1}, x_{3}\right)$-plane) owing to the hydrodynamic 


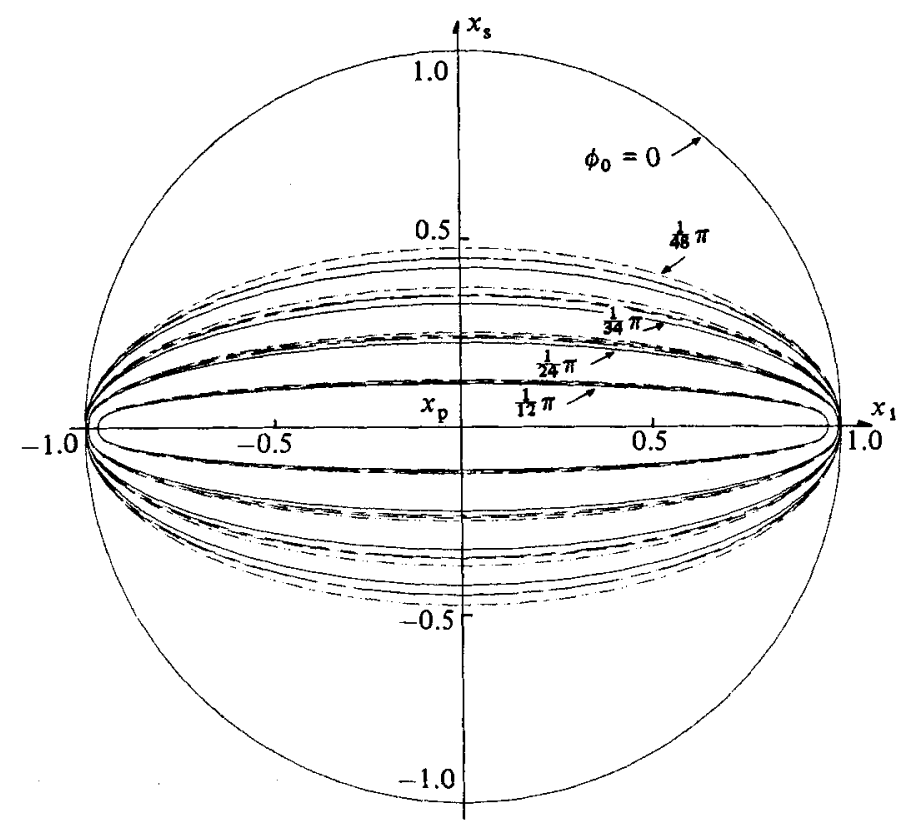

Figure 15. Orbital trajectories as obtained by the projection of the end of the particle onto the plane of the shear flow $\hat{O}_{2}=\Gamma_{13} x_{3} e_{1} ; \phi_{0}=0^{\circ}, \frac{1}{48} \pi, \frac{1}{36} \pi, \frac{1}{24} \pi$ and $\frac{1}{12} \pi, \theta_{0}=0^{\circ}, \kappa=20, S(x)=0$,

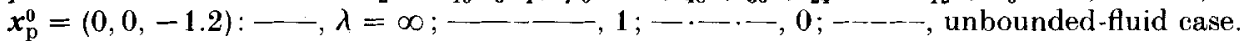

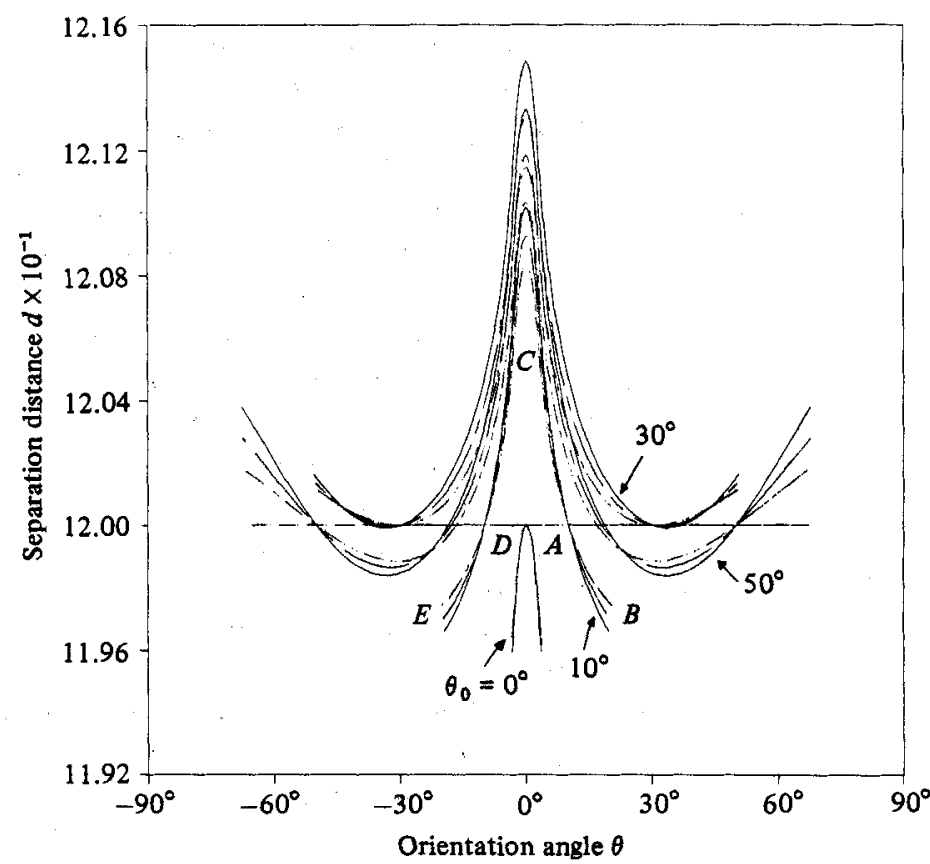

Figure 16. Trajectories for a neutrally buoyant slender body in a simple shear flow $O_{2}=\Gamma_{13} x_{3} e_{1}$ in terms of the separation distance $d$ and the orientation angle $\theta ; \phi_{0}=30^{\circ}, \theta_{0}=-30^{\circ}, 0^{\circ}, 10^{\circ}, 50^{\circ}$, $\kappa=20, S(x)=0, x_{p}^{0}=(0,0,-1.2):--, \lambda=\infty ;---, 1 ;-.-., \lambda=0 ;-\square$, unbounded-fluid case. 
force and torque, $F-K_{\mathrm{C}}^{\mathrm{t}} \cdot K_{\mathrm{R}}^{-1} \cdot T$ and $T-K_{\mathrm{C}} \cdot K_{\mathrm{T}}^{-1} \cdot \boldsymbol{F}$ in $(33 a, b)$. A detailed calculation shows that the twisting motion (i.e. rotation with $\dot{\phi}$ ) is enhanced by the presence of an interface, which tends to reduce the parallel translation of the nearest end to the interface and yields additional hydrodynamic torque on the body to increase $\dot{\phi}$.

We have also examined the trajectories of a slender body with $\phi_{0}=60^{\circ}$ and $90^{\circ}$. However, the qualitative features of the trajectories $(d$ versus $\theta$ ) for these cases are quite similar to the case of $\phi_{0}=30^{\circ}$, and illustrative figures are not necessary.

This completes our illustrative trajectory calculations for a neutrally buoyant particle (i.e. sphere or slender body) freely suspended in a pure straining or in a simple shearing flow, using the basic solutions that were developed in $\$ \S 3$ and 4 . In future research we plan to consider the application of the results of this paper to particle capture at the surface of a large bubble or drop (i.e. capture rates in flotation processes), and to the rheology of dilute suspensions.

This work was supported by a grant from the National Science Foundation, Fluid Mechanics Program of the Engineering Directorate. The authors are grateful for this support.

\section{REFERENCES}

BATCHELOR, G. K. 1970 Slender-body theory for particles of arbitrary cross-section in Stokes flow. J. Fluid Mech. 44, 419.

BrenNer, H. 1961 The slow motion of a sphere through a viscous fluid towards a plane surface. Chem. Engng Sci. 16, 242.

Chwang, A. T. \& WU, T. Y-T. 1975 Hydrodynamics of low-Reynolds-number flow. Part 2. Singularity method for Stokes flows. J. Fluid Mech. 67, 787.

Cox, R. G. 1971 The motion of long slender bodies in a viscous fluid. Part 2. Shear flow, J. Fluid Mech. 45, 625.

Darabaner, C. L. \& Mason, S. G. 1967 Particle motions in sheared suspensions. XXII: interactions of rigid sphere. Rheol. Acta 6, 273.

Dukhin, S. S. \& Rulev, N. N. 1977 Hydrodynamic interaction between a solid spherical particle and a bubble in the elementary act of flotation. Colloid J. USSR 39, 270.

FAXÉN, H. 1921 Dissertation, Uppsala University.

FULFORD, G. R. \& BLAKE, J. R. 1983 On the motion of a slender body near an interface between two immiscible liquids at very low Reynolds number. J. Fluid Mech. 127, 203.

Goldman, A. J., Cox, R. G. \& Brenner, H. 1967 a Slow viscous motion of a sphere parallel to a plane wall. I. Motion through a quiescent fluid. Chem. Engng Sci. 22, 637.

Goldman, A. J., Cox, R. G. \& Brenner, H. $1967 b$ Slow viscous motion of a sphere parallel to a plane wall. II. Couette flow. Chem. Engng Sci. 22, 653.

GoREN, S. L. \& O'NeILL, M. E. 1971 On the hydrodynamic resistance to a particle of a dilute suspension when in the neighborhood of a large obstacle. Chem. Engng Sci. 26, 325.

JEFEERY, G. B. 1912 On a form of the solution of Laplace's equation suitable for problems relating to two spheres. Proc. R. Soc. Lond. A 87, 109.

JefFery, G. B. 1922 The motion of ellipsoidal particles immersed in a viscous fluid. Proc. R. Soc. Lond. A 102,161

LEAL, L. G. 1975 The slow motion of slender rod-like particles in a second-order fluid. J. Fluid Mech. 69, 305.

Lee, S. H., Chadwick, R. S. \& Leal, L. G. 1979 Motion of a sphere in the presence of a plane interface. Part 1. An approximate solution by generalization of the method of Lorentz. J. Fluid Mech. 93, 705.

LeE, S. H. \& LeaL, L. G. 1980 Motion of a sphere in the presence of a plane interface. Part 2. An exact solution in bipolar coordinates. J. Fluid Mech. 98, 193.

Lorentz, H. A. 1907 A general theory concerning the motion of a viscous fluid. Abhandl. Theor. Phys. 1, 23. 
Spizlman, L. A. 1977 Particle capture from low-speed laminar flows. Ann. Rev. Fluid Mech. 9, 297.

WaKIYA, S. 1957 Viscous flows past a spheroid. J. Phys. Soc. Japan 12, 1130.

YANa, S.-M. \& LEAL, L. G. 1983 Particle motion in Stokes flow near a plane fluid-fluid interface. Part 1. Slender body in a quiescent fluid. J. Fluid Mech. 136, 393. 